\title{
Human mesenchymal stromal cells broadly modulate high glucose-induced inflammatory responses of renal proximal tubular cell monolayers
}

Md Nahidul Islam ${ }^{1}$, Tomás P. Griffin ${ }^{1,2}$, Elizabeth Sander ${ }^{1}$, Stephanie Rocks ${ }^{1}$, Junaid Qazi ${ }^{1}$, Joana Cabral ${ }^{1}$, Jasmin McCaul ${ }^{3}$, Tara McMorrow ${ }^{3}$ and Matthew D. Griffin ${ }^{1 *}$ (iD

\begin{abstract}
Background: Renal proximal tubular epithelial cells (RPTEC) are dysfunctional in diabetic kidney disease (DKD). Mesenchymal stromal cells (MSC) may modulate DKD pathogenesis through anti-inflammatory mediators. This study aimed to investigate the pro-inflammatory effect of extended exposure to high glucose (HG) concentration on stable RPTEC monolayers and the influence of MSC on this response.

Methods: Morphologically stable human RPTEC/TERT1 cell monolayers were exposed to $5 \mathrm{mM}$ and $30 \mathrm{mM}$ (HG) Dglucose or to $5 \mathrm{mM} \mathrm{D-glucose}+25 \mathrm{mM}$ D-mannitol (MAN) for 5 days with sequential immunoassays of supernatants and end-point transcriptomic analysis by RNA sequencing. Under the same conditions, MSC-conditioned media (MSC-CM) or MSC-containing transwells were added for days 4-5. Effects of CM from HG- and MAN-exposed RPTEC/MSC cocultures on cytokine secretion by monocyte-derived macrophages were determined.

Results: After 72-80 h, HG resulted in increased RPTEC/TERT1 release of interleukin (IL)-6, IL-8, monocyte chemoattractant protein (MCP)-1 and neutrophil gelatinase-associated lipocalin (NGAL). The HG pro-inflammatory effect was attenuated by concentrated (10X) MSC-CM and, to a greater extent, by MSC transwell co-culture. Bioinformatics analysis of RNA sequencing data confirmed a predominant effect of HG on inflammation-related mediators and biological processes/ KEGG pathways in RPTEC/TERT1 stable monolayers as well as the non-contact-dependent anti-inflammatory effect of MSC. Finally, CM from HG-exposed RPTEC/MSC transwell co-cultures was associated with attenuated secretion of inflammatory mediators by macrophages compared to CM from HG-stimulated RPTEC alone.

Conclusions: Stable RPTEC monolayers demonstrate delayed pro-inflammatory response to HG that is attenuated by close proximity to human MSC. In DKD, this MSC effect has potential to modulate hyperglycemia-associated RPTEC/ macrophage cross-talk.
\end{abstract}

\section{Background}

Diabetic kidney disease (DKD) is the leading cause of end stage renal disease (ESRD) worldwide [1]. The complex pro-inflammatory milieu of hyperglycaemia, reactive oxygen species (ROS), advanced glycation end products (AGE) and angiotensin-II contributes to activation of

\footnotetext{
* Correspondence: matthew.griffin@nuigalway.ie

${ }^{1}$ Regenerative Medicine Institute (REMEDI) at CÚRAM Centre for Research in Medical Devices, School of Medicine, National University of Ireland Galway, Galway, REMEDI, Biomedical Sciences, Corrib Village, Dangan, Galway H91 TK33, Ireland

Full list of author information is available at the end of the article
}

transcription factors, growth factors, inflammatory cytokines and chemokines that mediate glomerular, microvascular and tubulo-interstitial injury-eventually leading to progression to ESRD and to the increased cardiovascular mortality associated with DKD [2-4].

A substantial body of research evidence documents the links between chronic inflammation and the development and progression of DKD [5-8]. Hyperglycaemia induces cytokine production by macrophages and other immune cells which may serve both as drivers and predictive biomarkers for progressive loss of renal function

(C) The Author(s). 2019 Open Access This article is distributed under the terms of the Creative Commons Attribution 4.0 International License (http://creativecommons.org/licenses/by/4.0/), which permits unrestricted use, distribution, and 
$[7,9,10]$. For example, circulating concentration of monocyte chemoattractant protein-1 (MCP-1/CCL2) has been shown to correlate with the degree of interstitial macrophage infiltration in human DKD while, experimentally, inhibition of MCP-1 in models of diabetes mellitus (DM) ameliorates renal injury [11-13]. Hyperglycaemia upregulates MCP-1 production by kidney tubular epithelial cells, leading to infiltration of monocytes into the kidneys where they may subsequently become differentiated into inflammatory macrophages $[14,15]$. This is further associated with localised release of pro-inflammatory cytokines such as interleukin (IL)-1 $\beta$, IL-6 and tumour necrosis factor-alpha (TNF $\alpha)[15,16]$.

The renal proximal tubular epithelial cell (RPTEC) is a significant target for the adverse effects of chronic hyperglycaemia. Excessive glucose in the glomerular filtrate drives increased glucose reabsorption in the proximal tubules and activates a range of maladaptive pathways within RPTEC that contribute to the DKD pathogenesis [17-22]. As DKD progresses, secondary mediators including growth factors, angiotensin-II and AGE activate inflammatory signalling pathways to further increase ROS production, inflammation, tubular cell hypertrophy and interstitial fibrosis [1719, 21]. These insights highlight RPTEC as a potentially important therapeutic target in DKD, and in vitro studies involving cultured RPTEC-like cells provide a valuable testbed for identifying and evaluating novel interventional strategies [21, 23]. Among the in vitro tools available, RPTEC/TERT1 is an immortalised RPTEC cell line generated by overexpression of human telomerase reverse transcriptase (hTERT) [24]. Recent studies have highlighted the potential advantages of RPTEC/TERT1 stable monolayer cultures over other cell lines for modelling renal proximal tubular function and responses [25-27].

Macrophages are key mediators of intra-renal inflammation in DM, being an important source of proinflammatory factors including IL-1, TNF $\alpha$, IL-6 and ROS [28]. In vivo, macrophage infiltration and activation within the kidneys of diabetic animals as well as other models of renal injury has been shown to contribute significantly to increased production of chemokines, interstitial fibrosis and increased serum creatinine and proteinuria [29-32]. Combined with the direct effects of chronic hyperglycaemia to induce pro-inflammatory responses in RPTEC, these studies indicate that crosstalk between RPTEC and interstitial macrophages within the kidney represents a key pathological axis in the development and progression of DKD.

Currently, a limited number of therapies are available that specifically target the development and progression of DKD. Novel interventions that modulate multiple inflammatory pathways as well as promote repair of tubuleinterstitial injury could well complement conventional drug classes that predominantly address maladaptive glomerular pathophysiology in DKD. Relevant to this, interventions to inhibit pro-inflammatory cross-talk between RPTEC and macrophages represent an attractive strategy. Mesenchymal stem/stromal cell (MSC) therapy is a potential therapeutic option for diverse inflammatory disease pathologies [2, 33, 34]. In their physiological, perivascular niches in the bone marrow and other tissues, MSC have critical roles in immunomodulation and self-renewal [2, 34, 35]. Recent studies in animal models of DKD indicate that systemic administration of MSC ameliorates DM-associated albuminuria and renal pathological abnormalities in a paracrine manner through immunomodulatory and anti-apoptotic effects [36-39]. The potential clinical translation of MSC therapy for progressive DKD has reached the stage of early-phase clinical trials but the precise mechanisms of action of MSC remain incompletely characterised.

In this study, we aimed to determine the immunological consequences of prolonged exposure of RPTEC/TERT1 stable monolayers to high concentrations of glucose and to investigate the modulatory effects of culture-expanded human MSC and their soluble products on high glucose (HG)-induced RPTEC inflammatory response and the resulting RPTEC/macrophage cross-talk.

\section{Methods \\ RPTEC/TERT1 cell culture and treatments}

RPTEC/TERT1 (human renal proximal tubular epithelial cell line from the American Tissue Culture Collection) were cultured in 24-well flat-bottom plates (Sarstedt, Numbrecht, Germany) in Dulbecco's modified Eagle's medium (DMEM) (Gibco, Grand Island, NY, USA) and Ham's F-12 medium (Gibco) at 1:1 supplemented with ITS (Sigma Aldrich, St. Louis, MO, USA) containing $10 \mu \mathrm{g} / \mathrm{ml}$ insulin, $5.5 \mu \mathrm{g} / \mathrm{ml}$ transferrin and $5 \mathrm{ng} / \mathrm{ml}$ sodium selenite; $10 \mathrm{ng} / \mathrm{ml}$ epidermal growth factor (Sigma); $36 \mathrm{ng} /$ $\mathrm{ml}$ hydrocortisone (Sigma); $2 \mathrm{mM}$ L-glutamine (Gibco) and $100 \mathrm{U} / \mathrm{ml}$ penicillin and $100 \mu \mathrm{g} / \mathrm{ml}$ streptomycin (Gibco) and maintained at $37^{\circ} \mathrm{C}, 5 \% \mathrm{CO}_{2}$ in a humidified tissue culture incubator. For stabilisation of the monolayer, 27,500 cells $/ \mathrm{cm}^{2}$ were plated, cultured for 6 days to $100 \%$ confluency then allowed to form stable monolayers for a further 6 days before use in individual experiments. The medium was replaced every 2 days. For "high glucose (HG)" and "mannitol osmotic control (MAN)" culture conditions, the medium was additionally supplemented with $25 \mathrm{mM}$ D-glucose (Sigma) or $25 \mathrm{mM} \mathrm{D-mannitol}$ (Sigma) respectively was added at day 12 and maintained for a further 4-5 days. In some experiments, the medium was also supplemented with $100 \mu \mathrm{g} / \mathrm{ml}$ human serum albumin (Sigma), or $1 \mathrm{ng} / \mathrm{ml}$ IL-1 $\beta$ (Peprotech EC Ltd., NJ, USA) and $20 \mathrm{ng} / \mathrm{ml} \mathrm{TNF} \alpha$ (Peprotech) for the final 5 or 2 days of culture respectively. Phase-contrast microscopy and image capture of cultured cells were performed at intervals using an Olympus-IX71 inverted microscope 
(Tokyo, Japan). Osmolality of the cell culture supernatants from CTRL, HG and MAN conditions was measured in the Clinical Biochemistry Laboratory, Galway University Hospitals.

Culture of mesenchymal stromal cells and control cells Cryopreserved human bone marrow-derived MSC (BMMSC) from two healthy donors were cultured in MEMAlpha media (Gibco) supplemented with $10 \%$ extracellular vesicle (EV)-free heat-inactivated foetal calf serum (FCS) (Gibco), 1\% penicillin/streptomycin (Gibco) and $1 \mathrm{ng} / \mathrm{ml}$ fibroblast growth factor (R\&D Systems, Minneapolis, MN, USA). EV-free FCS was prepared by ultracentrifugation of FCS at 100,000 $\times g$ (Sorvall 100SE Ultra Centrifuge) for 18 $\mathrm{h}$ and subsequent collection of supernatants. Culture of human corneal endothelial cells (HCEC) was carried out in DMEM supplemented with 10\% FCS (Gibco) and 1\% penicillin/streptomycin (Gibco). Conditioned media were prepared as described in Additional file 1: Supplementary Methods. In the case of the MSC-derived CM, this was further divided into non-manipulated CM ("MSC-CM (Whole)") and MSC-CM from which the MSC-derived $\mathrm{EV}$ were depleted by ultracentrifugation for $18 \mathrm{~h}$ as 100 , $000 \times g$ ("MSC-CM (-EV)").

\section{Indirect co-culture of RPTEC/TERT1 cells and mesenchymal stromal cells}

RPTEC/TERT1 cells were plated at $27,500 \mathrm{cells} / \mathrm{cm}^{2}$ in six-well tissue culture plates and were cultured for 12 days to form stable monolayers. The cells were then cultured in medium additionally supplemented with $25 \mathrm{mM}$ D-glucose (Sigma) or D-mannitol (Sigma) for a further 5 days as described above. Human BM-MSC were separately seeded at 10,000 cells $/ \mathrm{cm}^{2}$ into transwell inserts (ThinCert ${ }^{\mathrm{tw}}$, Greiner Bio-One, Kremsmünster, Austria) for 3 days in MSC culture medium. On day 15 of RPTEC/TERT1 cell culture, the BM-MSC-containing inserts were placed on top of individual RPTEC/TERT1 monolayer-containing wells and these co-cultures were maintained for a further 2 days, following which the RPTEC/TERT1 cell pellets and supernatants were collected for protein analysis and enzyme-linked immunosorbent assays (ELISA) respectively. Transwell cocultures of RPTEC/TERT1 cells and HCEC were carried out by the same protocol.

\section{Enzyme-linked immunosorbent assays}

Assay kits for Interleukin-1 $\beta$ (IL-1 $\beta$ ), IL-6, IL-8, TNF $\alpha$, IL10, MCP-1 and neutrophil gelatinase-associated lipocalin (NGAL) (R\&D Systems, MN, USA) were used to perform ELISAs of culture supernatants according to the manufacturer's instructions (see Additional file 1: Supplementary Methods for a detailed protocol).

\section{Flow cytometry}

Viability of RPTEC/TERT1 cells was determined by propidium iodide (PI) (Molecular Probes, Oregon, USA) staining. In brief, following trypsinization and centrifugation, cell pellets were re-suspended in culture medium and incubated for $15 \mathrm{~min}$ at $37^{\circ} \mathrm{C}$ to restore membrane integrity. The cell suspensions were then washed and re-suspended in FACS buffer containing 2\% FCS (Gibco) and $0.05 \% \mathrm{NaN}_{2}$ (Sigma) in PBS (Sigma). Cells were transferred as $100-\mu \mathrm{l}$ aliquots into 5-ml polystyrene FACS tubes (Sarstedt). Finally, PI solution was added to final concentration of $1 \mu \mathrm{g} / \mathrm{ml}$ and the samples were analysed on an Accuri-C6 flow cytometer (Becton Dickinson, USA) using CFlow software.

\section{Western blotting}

Immunoblots of RPTEC-derived protein lysates for NF-kB p65, phospho-NF-kB p65 (pP65), p38 MAPK, phospho-p38 MAPK (pP38MAPK), p44/42 MAPK (Erk1/2), phosphop44/42 MAPK (pErk1/2; Thr202/Tyr204), STAT1, phospho-Stat1 (pSTAT1; Tyr701), protein kinase-C alpha (PKC $\alpha)$, phospho-PKC $\alpha / \beta$ II (pPKC $\alpha$; Thr638/641) and PPAR $-\gamma$ were performed using reagents and procedures described in Additional file 1: Supplementary Methods.

\section{RNA isolation and quantification}

Total RNA was isolated from RPTEC/TERT1 cells using TRIzol/Chloroform method and by RNEasy Midi Kit (Qiagen, Hilden, Germany). A detailed protocol for RNA isolation by TRIzol method is provided in Additional file 1: Supplementary Methods. For samples prepared using RNEasy Midi Kit (Qiagen), the manufacturer's recommended protocol was followed. The quality and integrity of all RNA samples were measured by Bioanalyzer-2100 using RNA 6000 Pico kit (Agilent Technologies) according to the manufacturer's recommended protocol.

\section{RNA sequencing and bioinformatics analysis}

High-throughput RNA sequencing (RNA-seq) was performed by BGI Genomics Service (Hong Kong) using BGISEQ-500, and Bioinformatics analyses of the resulting transcriptional profiles were performed using a suite of software packages including RSEM (quantitation of gene expression level), Cluster and Java Treeview Cluster (clustering analysis of gene expressions), Medusa (protein-protein interactions), WCGNA and Cytoscape (gene coexpression network analysis). Only RNA samples with RNA integrity number (RIN) $\geq 7.0$ were subjected to RNA-seq. In brief, following fragmentation of mRNA and subsequent reverse transcription and amplification, a sequencing library was prepared. Nucleotide sequence of the fragments was determined and high-quality reads were aligned to the reference genomic sequence. Fragments that matched the genomic sequence were assigned to a specific position of a specific chromosome in the genome; 
thereby, the gene fragments could be linked to a specific gene. The number of reads per gene were counted and normalised. The criteria for designation of differentially expressed genes (DEG) were $>1.5$ absolute fold-change and statistical significance $(p<0.05)$ among experimental conditions. RNA-seq data analyses involved plotting data using principal component analysis and KEGG pathway enrichment analysis.

\section{Quantitative, reverse transcription polymerase chain reaction (qRT-PCR)}

For qRT-PCR, the Luna Universal Probe One Step RTqPCR kit (New England BioLabs, MA, USA) was used according to the manufacturer's instructions. Reactions consisted of $50 \mathrm{ng}$ RNA samples, mastermix, nuclease-free water, enzyme and primer/probe in a final volume of $10 \mu \mathrm{l}$. Individual target specific primers (both forward and reverse; for IL-6, MCP-1, IL-1 $\beta$, IL-8, TNF $\alpha$, NGAL and RPLP0) and TaqMan probes (for quantitation) were purchased from Integrated DNA Technologies (Coralville, Iowa, USA). The primer sequences are listed in Additional file 2: Table S1. The reactions were performed on Step-One Plus PCR instrument (Applied Biosystems, Waltham, MA, USA). Mean Ct values were used to calculate the fold changes in the expression of different target genes (for IL-6, MCP-1, IL-1 $\beta$, IL-8, TNF $\alpha$ and NGAL) in treatment groups vs. control as determined relative to the housekeeping gene RPLP0 using the $2^{-\Delta \Delta C t}$ method.

\section{Culture of primary human macrophages and exposure to conditioned media}

Human peripheral blood mononuclear cells (PBMCs) were prepared and cultured overnight using a standard protocol (described in detail in Additional file 1: Supplementary Methods). Plastic-adherent PBMCs were cultured in 24-well plates (Sarstedt) at a density of 22,500 cells $/ \mathrm{cm}^{2}$ in macrophage medium containing $20 \mathrm{ng} / \mathrm{ml}$ granulocyte macrophage colony stimulating factor (GM-CSF, Peprotech). Medium was replaced every 3 days. After 9 days, the medium was replaced with conditioned medium (CM) from the RPTEC/TERT1 co-culture experiments with addition of GM-CSF to a final concentration of $20 \mathrm{ng} / \mathrm{ml}$. To some wells, $100 \mathrm{ng} / \mathrm{ml}$ interferon gamma (IFN , Peprotech), $100 \mathrm{ng} / \mathrm{ml}$ TNF $\alpha$ (Peprotech) and $50 \mathrm{ng} / \mathrm{ml}$ LPS (Sigma) were added to provide a positive control for pro-inflammatory stimulation. After $24 \mathrm{~h}$, the CM was removed, the macrophages were washed with PBS and fresh macrophage medium containing GM-CSF was added. Finally, after an additional $24 \mathrm{~h}$ of culture, the supernatants were collected for subsequent analysis by ELISA.

Statistical analysis was performed using GraphPad Prism version 6.0. Paired- or unpaired Student's $t$ test, non-parametric multiple $t$ test and one- or two-way ANOVA were used for analysis of individual experiments as appropriate. Details of statistical analyses performed for specific experiments are provided in individual figure legends. For all statistical analyses, the threshold for significance was $<0.05$. Experiments were performed at least three times unless otherwise stated in the figure legends.

\section{Results \\ Prolonged exposure to high glucose enhances inflammatory response of RPTEC/TERT1 monolayers}

Based on previously published characterizations of the RPTEC/TERT1 cell line by us and others [25-27], culture conditions were established under which RPTEC-TERT1 cells, seeded at an optimised initial density (Additional file 3: Figure S1A), reached confluence by day 6 and formed stable monolayers by day 12 . As shown in Fig. 1a, seeded cells progressed from sub-confluent, spindle-shaped cells to confluent layers of tightly packed cells of cobblestone appearance between days 0 and 6 of culture then developed frequent dome-shaped protuberances between days 8 and 12. After 12 days of culture, RPTEC/TERT1 monolayers were cultured for an additional 4 days under RM, HG or MAN conditions. As shown in Fig. 1b, RPTEC/TERT1 monolayers continued to show a confluent, cobblestone appearance during this time period and no morphological differences were observed among the culture conditions. The osmolalities of HG and MAN culture media were confirmed to be higher than that of the basal medium ( $\mathrm{HG}=360 \pm 10 \mathrm{mosm} / \mathrm{ml}$; $\mathrm{MAN}=366 \pm 9 \mathrm{mosm} / \mathrm{ml} ; \mathrm{CTRL}=339 \pm 3 \mathrm{mosm} / \mathrm{ml}$ ).

The influence of high glucose (HG) exposure for a further 5 days $(120 \mathrm{~h})$ on secretion of inflammation-related soluble factors (IL-6, IL-8 and MCP-1) and tubular injuryrelated marker NGAL into the culture medium was then compared to that of normal-glucose (CTRL) and mannitol osmotic control (MAN) culture conditions (Fig. 2a, b). As shown, increased secretion of all four analytes was observed under HG but not MAN conditions relative to CTRL between 80 and $120 \mathrm{~h}$ of culture. The HG-induced increases in inflammatory factors were not associated with increased cell death (Fig. 2c). As further evidence of the relevance of the culture system to diabetic conditions, it was verified that the exposure of RPTEC/TERT1 monolayers to other relevant stimuli-human serum albumin (HSA) and IL$1 \beta-$ known to exert pro-inflammatory effects on proximal tubular epithelial cells, resulted in increased secretion of IL6, IL- 8, MCP-1 and NGAL that was additive to the induction associated with HG culture (see Additional file 4: Figure S2 and Additional file 5: Figure S3). Neither HSA nor IL-1 $\beta$ were associated with increased cell death during culture (data not shown). Western blotting of RPTEC/TERT1 monolayer-derived protein lysates collected at 24,48 and $96 \mathrm{~h}$ following exposure to CTRL, HG and MAN conditions showed evidence of 


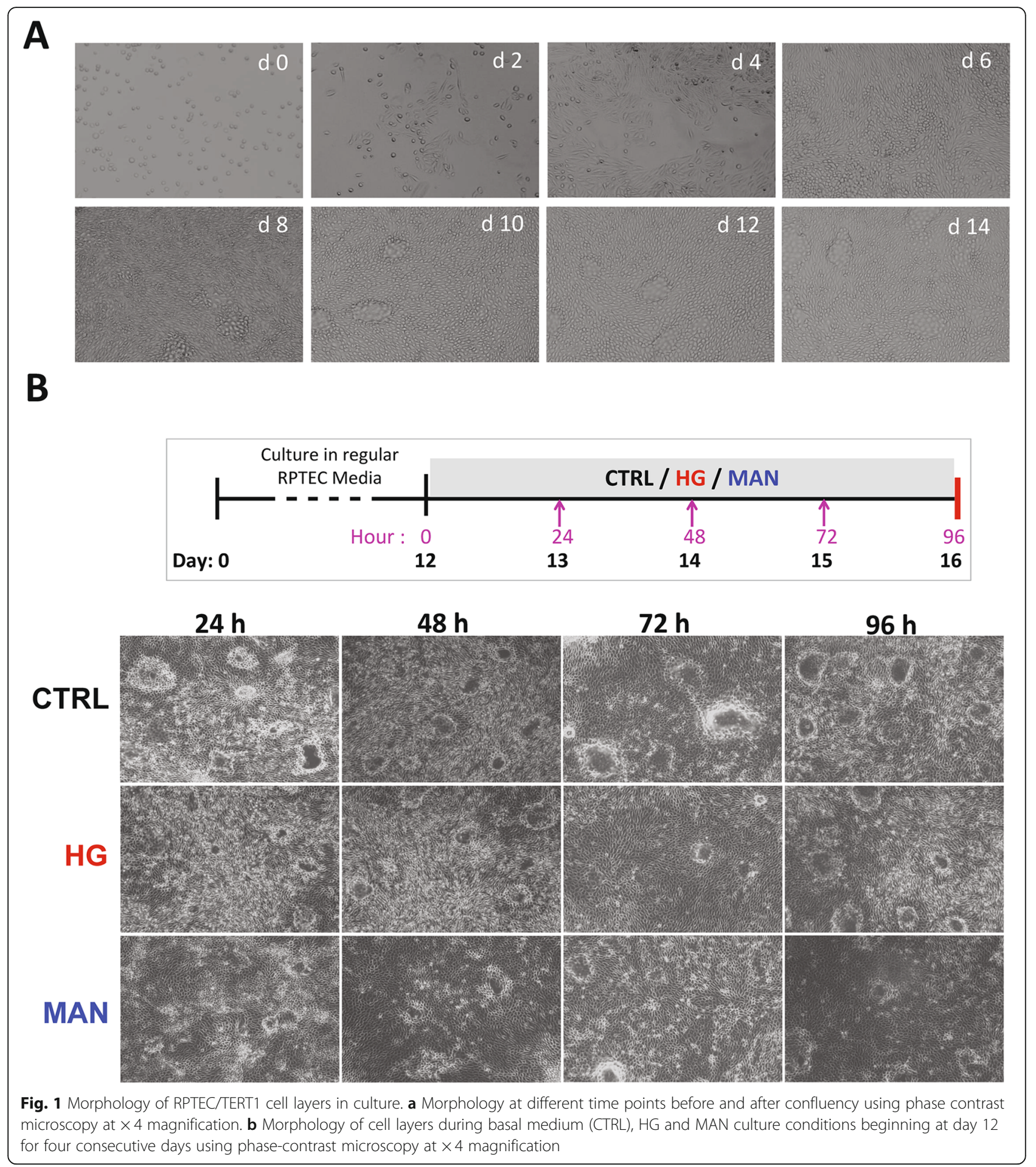

activation of multiple potentially pro-inflammatory intracellular signalling pathways (NF-kB, p38 and ERK1/2 MAPK and PKC $\alpha$ ). However, the level of activation, based on abundance of phosphorylated pathway components, was no greater for HG compared to the control conditions (see Additional file 6: Figure S4A and Additional file 7: Figure S4B). It was concluded that prolonged exposure of mature RPTEC/TERT1 cell monolayers to HG results in increased release of proinflammatory mediators that is not driven by sustained hyperactivity of the individual signalling pathways examined and is not associated with overt loss of viability, gross morphological changes or dedifferentiation to a non-epithelial phenotype. 

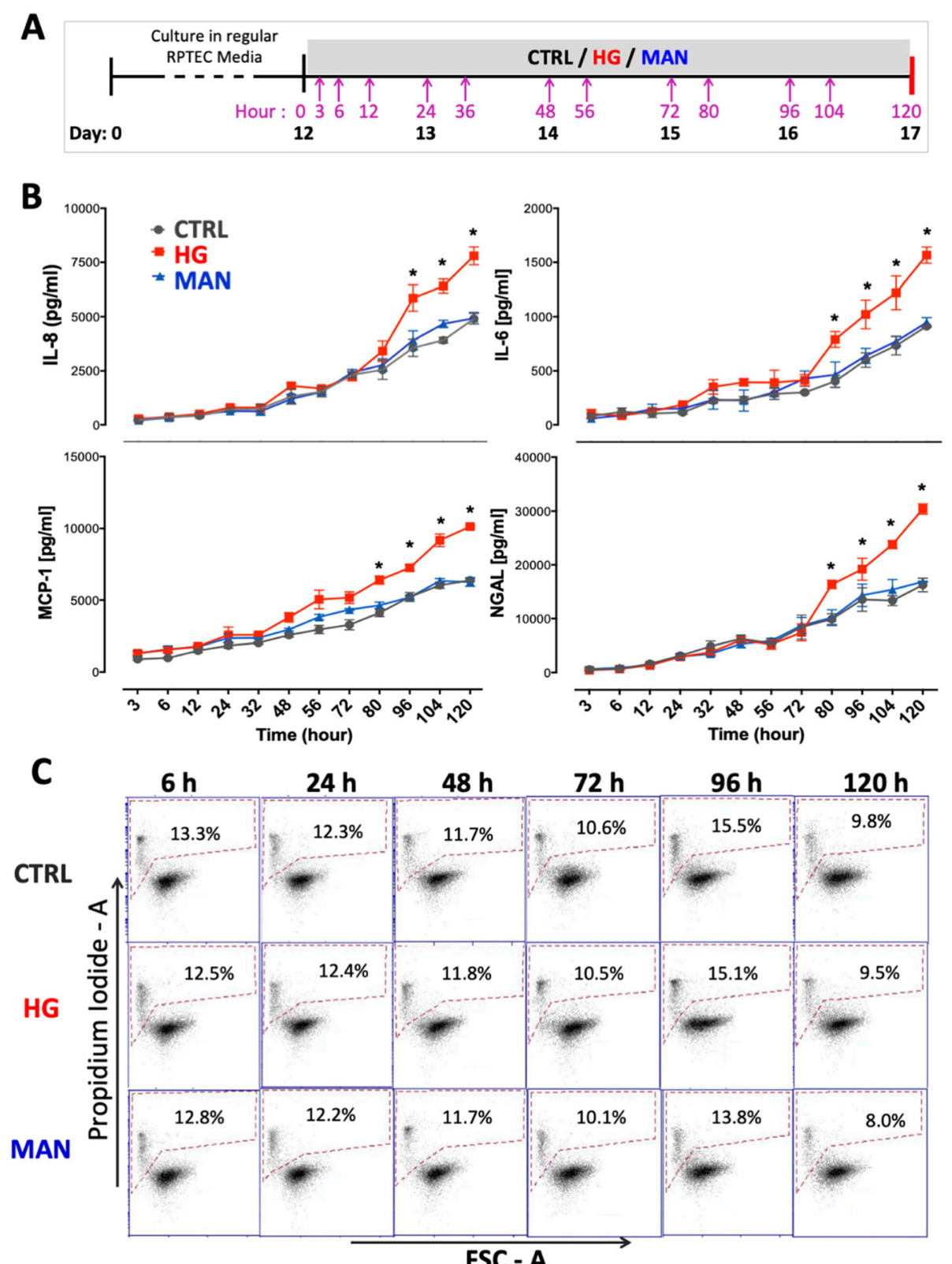

Fig. 2 High glucose exposure increases the inflammatory response of RPTEC/TERT1 cell stable monolayers: a Schematic diagram of the experimental protocol. b Concentrations of inflammatory biomarkers IL-8 (top left), IL-6 (top right), MCP-1 (bottom left) and tubular injury marker NGAL (bottom right) in culture supernatants of RPTEC/TERT1 cell stable monolayers between 3 and $120 \mathrm{~h}$ following exposure to medium containing normal (5 mM) glucose (CTRL, grey line), high (30 nM) glucose ( $\mathrm{HG}$, red line) and $5 \mathrm{mM}$ glucose $+25 \mathrm{mM}$ mannitol (MAN, blue line). All results are presented as mean \pm SD. Statistical analyses were performed by one-way ANOVA. ${ }^{*} p<0.05$. c Representative examples of flow cytometric analysis of RPTEC/TERT1 cell viability by propidium iodide exclusion at various time-points following exposure to the three culture conditions. Percentages of dead cells $\left(\mathrm{PI}^{+\mathrm{ve}}\right)$ are shown for each condition

Conditioned medium from human mesenchymal stromal cells inhibits the high glucose-induced inflammatory response of RPTEC/TERT1 monolayers

Early-passage human BM-MSC from healthy adult donors were shown to have expected surface marker profiles and osteogenic and adipogenic differentiation potential (see Additional file 8: Figure S5). To determine whether MSC-derived soluble products suppress RPTEC/TERT1 inflammatory responses, 10x-concentrated MSC-CM with and without depletion of extracellular vesicles (EVs) was added at $20 \%$ volume to RPTEC/ TERT1 monolayers for the final $48 \mathrm{~h}$ of 5 -day cultures in HG and MAN conditions (Fig. 3). Conditioned media of BM-MSC from two different donors were used. As 
shown, both MSC-CM preparations resulted in reduced secretion of IL-8, IL-6 and MCP-1 but not NGAL under HG and MAN conditions. Of note, the suppressive effect of MSC-CM on pro-inflammatory cytokines and chemokines was similar for CM preparations with and without EV depletion, suggesting that the effect was unlikely to be mediated by MSC-EV. In contrast, addition of CM from a non-MSC cell line (HCEC) resulted either in no reduction or in increased release of IL-6, IL-8, MCP-1 and NGAL by RPTEC/TERT1 monolayers.

\section{Indirect co-culture of human mesenchymal stromal cells causes a more potent inhibition of high glucose-induced inflammatory response of RPTEC/TERT1}

Next, it was determined, using a transwell co-culture system, whether indirect contact of BM-MSC diminishes the RPTEC/TERT1 inflammatory response to HG and control culture conditions. As shown in Fig. 4, indirect contact with BM-MSC from two different donors (but not indirect contact with HCEC) for the final 2 days of a 5 -day culture resulted in potent reductions of secretion of IL-8, IL- 6 and MCP-1 by HG-exposed RPTEC/TERT1 cell monolayers as well as more modest reductions under MAN-exposed conditions. In contrast to the effect of MSC-CM addition, transwell co-culture with MSC (as well as with HCEC) resulted in potent reduction of NGAL release by the monolayers under HG and, to a lesser extent, MAN conditions.

\section{Prolonged exposure of RPTEC/TERT1 monolayers to high glucose concentration is associated with widespread transcriptional modifications that are modulated by indirect co-culture with MSC}

To more broadly characterise the effect of prolonged HG exposure, RNA-seq was performed on triplicate samples of mature RPTEC/TERT1 monolayers following 5-day culture in CTRL, HG and MAN culture conditions (Fig. 5a). For this analysis, hierarchical clustering and principal component analyses demonstrated partial overlap between CTRL and MAN transcriptional profiles with separation of the HG profile from both controls (Fig. 5b, c). In total, 527 and 374 genes were differentially expressed in HG-exposed compared with CTRL- and MAN-exposed RPTEC/TERT1 monolayers respectively, with 115 (26 upregulated and 89 downregulated) DEGs being common to both comparisons (Fig. 5d). In contrast, only 314 DEG were identified for MAN compared to CTRL samples. Full lists of the DEGs from these comparisons are provided in Additional file 9: Table S2, Additional file 10: Table S3 and Additional file 11: Table S4. Biological process and pathway enrichment analysis of the DEGs from HG vs. MAN comparison indicated a predominance of processes and pathways related to inflammation and infection (see Additional file 12: Figure S6). Prominent among the modulated pathways were TNF- signalling pathway, cytokine-cytokine receptor interaction and NOD-like receptor signalling pathway. Among 15 DEGs within the TNF signalling pathway, multiple inflammationrelated transcripts were present including IL1- $\beta$, TNF, IL-6, L-selectin, colony stimulating factor 2 (CSF-2), IL-8, C-C motif chemokine ligand 2 (CCL2; also called MCP-1), lymphotoxin beta (LTB) and cellular inhibitor of apoptosis 1 and 2 (cIAP1/2) (see Additional file 13: Figure S5).

Next, a similar RNA-seq analysis was carried out on triplicates of RPTEC/TERT1 monolayers following 5-day exposure to $\mathrm{HG}$ and MAN conditions in the presence or absence of indirect (transwell) MSC co-culture for the final 2 days (Fig. 6a). As shown in Fig. 6b, principal component analyses demonstrated distinctive transcriptional profiles for the four conditions-indicating broad modulatory effects of indirect MSC co-culture on RPTEC/TERT1 gene expression under both HG and osmotic control conditions. In total, 811 genes were differentially expressed in MSC-co-cultured vs. nonMSC-(control)-cultured RPTEC/TERT1 monolayers under HG condition of which 395 were upregulated and 416 were downregulated. For the MAN condition, 916 genes were differentially expressed between non-MSC (control)-co-cultured vs. MSC-co-cultured cells of which 576 were upregulated and 368 were downregulated. A total of 281 genes (148 upregulated and 133 downregulated) were modulated by MSC co-culture under both HG and MAN conditions (Fig. 6c). Among the latter were multiple transcripts of relevance to inflammatory response including those encoding CCL2/MCP-1, chemokine (C-X-C motif) ligand 2 (CXCL2), enolase-2, interleukin 2 receptor subunit gamma (IL2RG), IL1- $\beta$, prostaglandin-endoperoxide synthase 1 (PTGS1), lipocalin 2 (LCN2; also called NGAL), S100 calcium binding protein A14 (S100A14), L-selectin (SELL) and superoxide dismutase 2 (SOD2) (Full lists of the DEGs from these comparisons are provided in Additional file 14: Table S5 and Additional file 15: Table S6).

Biological process (presented in Additional file 16: Figure S8) and pathway enrichment (Fig. 6d, e) analyses of the control-co-cultured vs. MSC-co-cultured RNA-seq profiles under HG and MAN conditions indicated prominent modulation of a range of inflammation-related responses. Notable among the pathways that were significantly modulated by indirect MSC co-culture in the presence of HG were TNF-signalling pathway, cytokine-cytokine receptor interaction, NOD-like receptor signalling pathway and arachidonic acid metabolism. Transcripts involved in these pathways included those encoding IL1 $\beta$, TNF, interleukin-6 (IL-6), IL18R, SELL, CSF2, TNF Alpha Induced Protein 3 (TNFAIP3), IL-8, CCL2, CXCL2, LTB and CIAP1/2 (Additional file 17: Figure $S 9$ provides a representative example). Quantitative RT-PCR analysis confirmed the RNA-seq results for 5 inflammation-related transcripts (Fig. 7).

Overall, it was concluded from the transcriptional profiling analyses of RPTEC/TERT1 monolayers that 

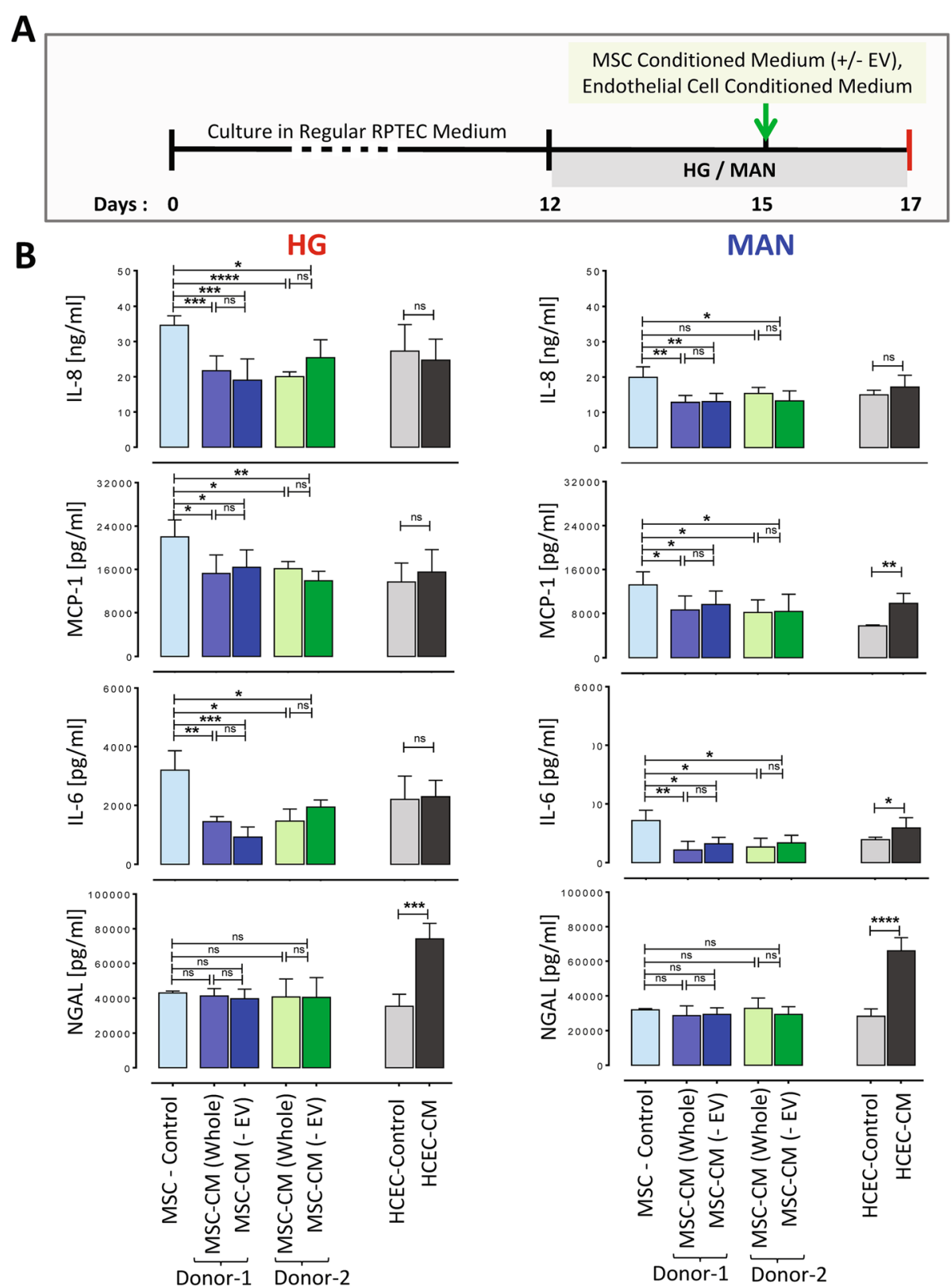

Fig. 3 Total and extracellular vesicle-depleted conditioned media from human MSC but not HCEC inhibit high glucose-induced secretion of inflammatory cytokines by RPTEC/TERT1 cell stable monolayers. a Schematic diagram of the experimental protocol $(+/-$ EV $=$ without and with extracellular vesicle depletion). b Graphs depicting the concentrations of IL-8, IL-6, MCP-1 and NGAL in the supernatants from RPTEC/TERT1 cell monolayers cultured without (control) and with various conditioned media (CM) during the final 2 days of 5-day cultures under high glucose (HG, left graphs) and mannitol (MAN, right graphs) conditions. All results are expressed as mean \pm SD of $n=6$ technical replicates for each condition. For cultures containing human (h) BM-MSC-derived CM, results are shown for MSC from two different donors [Donor 1 (blue) and Donor 2 (green)]. MSC-control = Unconditioned MSC medium. MSC-CM (whole) = Non-extracellular vesicle (EV) depleted MSC-CM. MSC-CM (-EV) = Extracellular vesicle (EV) depleted MSC-CM. HCEC-control = Unconditioned human corneal endothelial cell medium. HCEC-CM =CM from human corneal endothelial cells. Statistical analyses performed by one-way ANOVA (for hBM-MSC versus control) and unpaired Student's $t$ test (for endothelial cell versus control). ${ }^{* * *} p<0.0001,{ }^{* *} p<0.001,{ }^{*} p<0.05$, ns $=$ not significant

prolonged exposure to HG results in enhanced expression/ activity of a wide range of genes and pathways associated with immune response and inflammation and that proximity to viable human BM-MSC for $48 \mathrm{~h}$ exert a broad inhibitory effect on many of these inflammatory pathways-likely through the production of soluble mediators. Furthermore, the anti-inflammatory effect of MSC on proximal tubular epithelial monolayers is not exclusive to a HG-induced proinflammatory response, as it was also evident under MAN (osmotic control) conditions. 


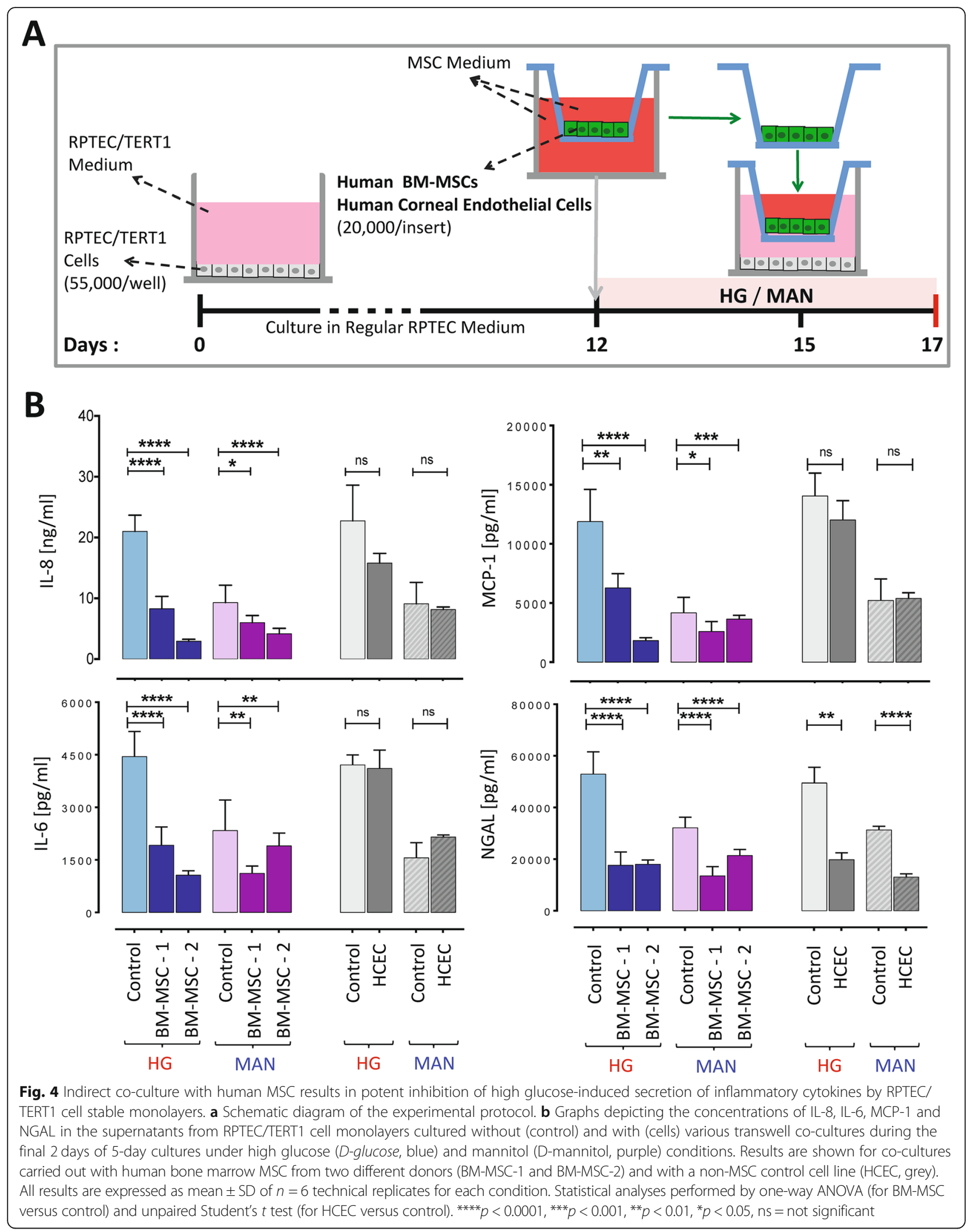




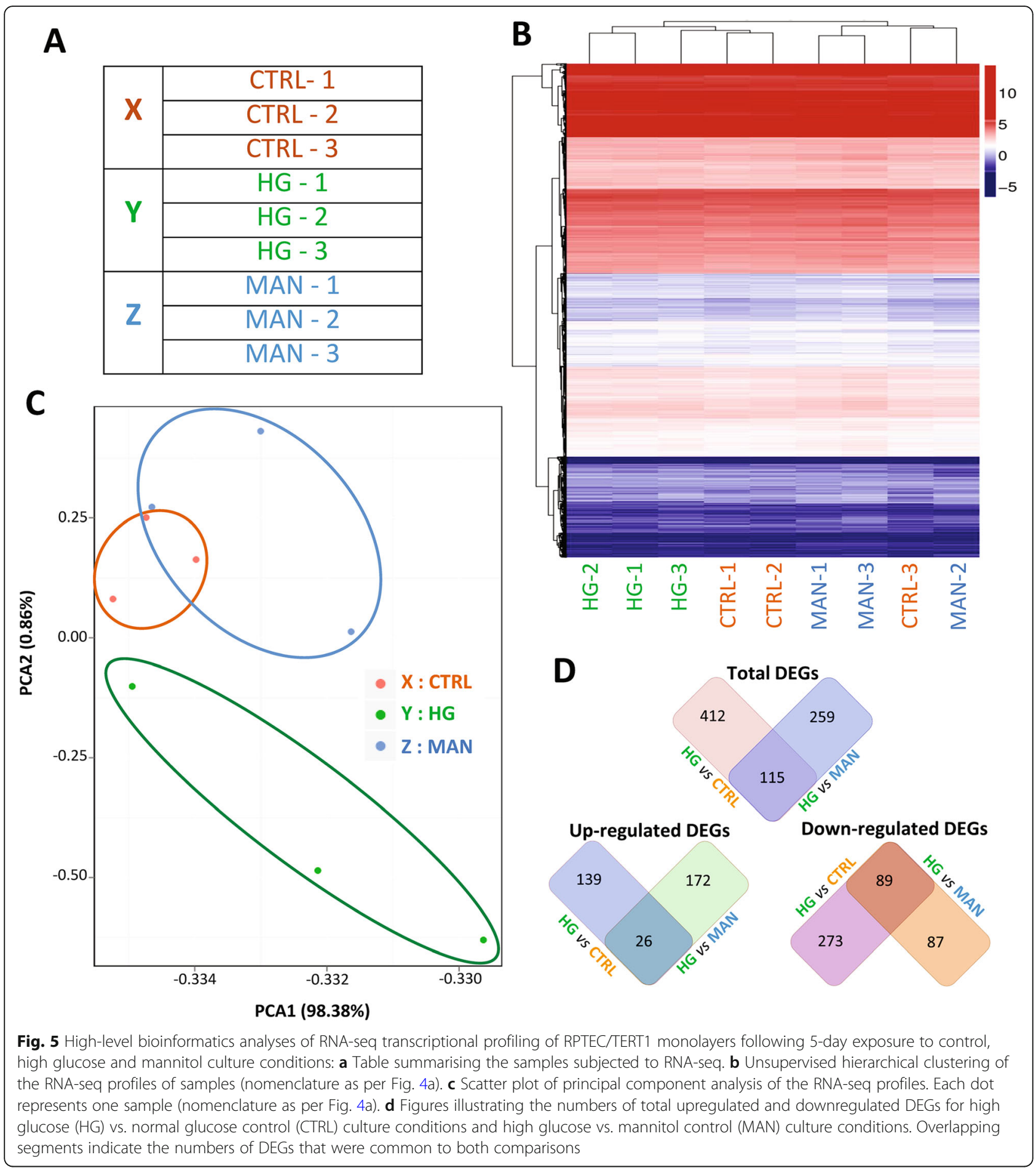

Indirect mesenchymal stromal cell contact modulates proinflammatory cross-talk between high glucose-exposed RPTEC/TERT1 cell monolayers and human macrophages Finally, we sought to determine whether soluble products released during RPTEC/TERT1-MSC co-cultures under HG or MAN conditions exert anti-inflammatory effects on human macrophages. An experiment was carried out in which in vitro-differentiated, human monocyte-derived macrophages were cultured for $24 \mathrm{~h}$ in the presence of CM from RPTEC/TERT1 monolayers that had been exposed for 5 days to HG or MAN conditions in the presence or absence of BM-MSC-containing transwells for the final 2 days (Fig. 8a). The CM-exposed macrophages were then washed and allowed to remain 


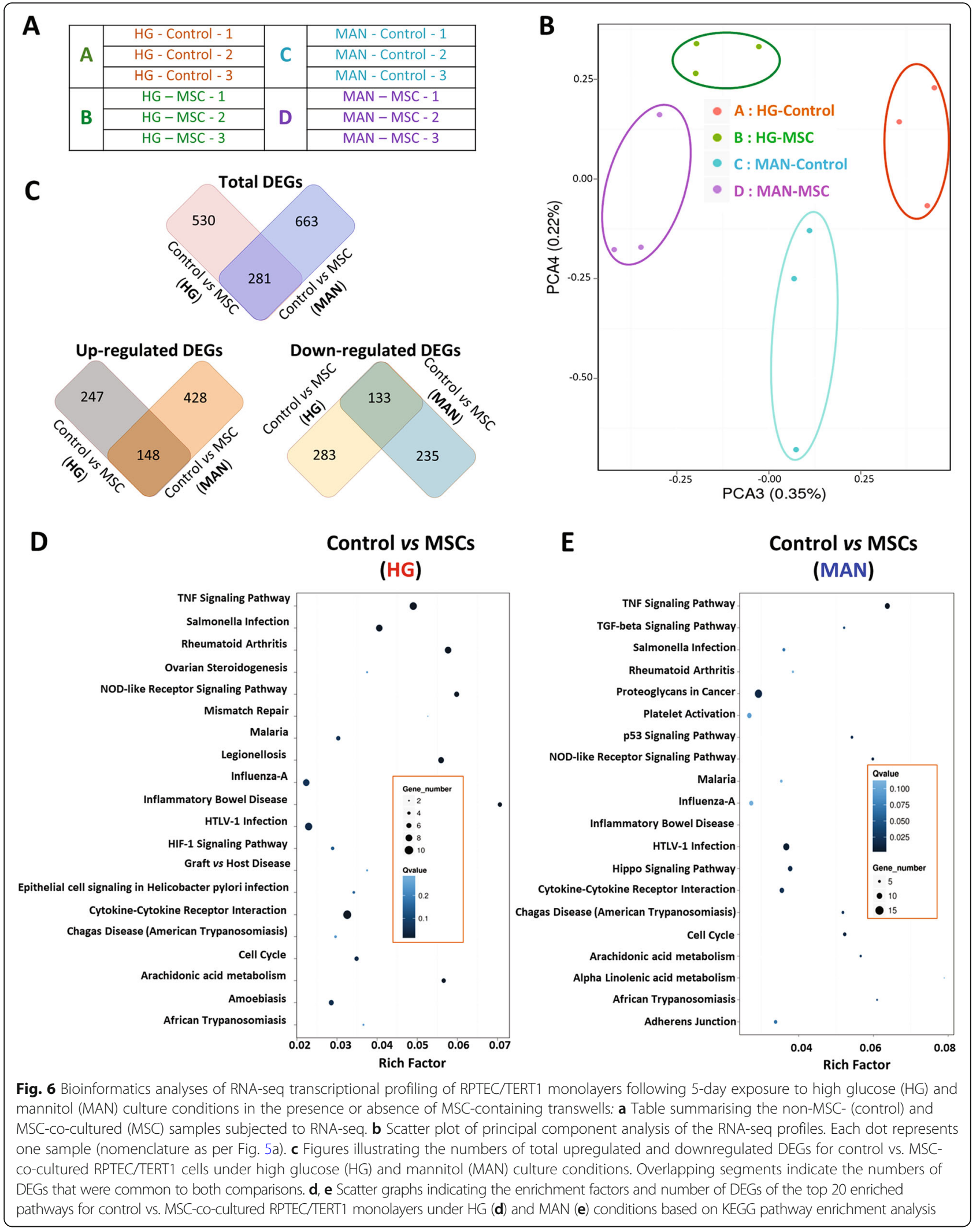




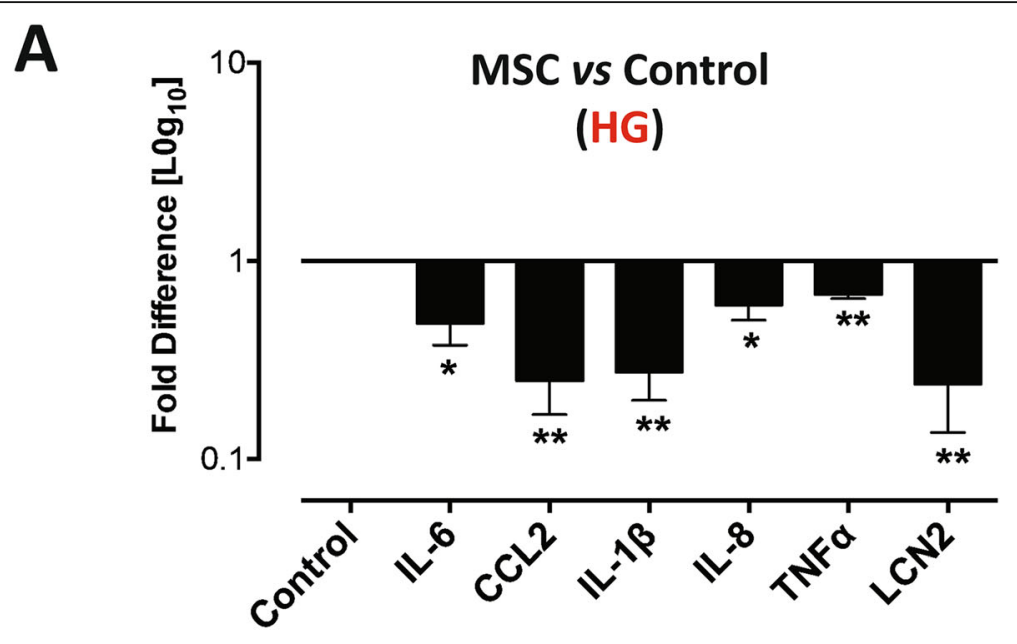

B

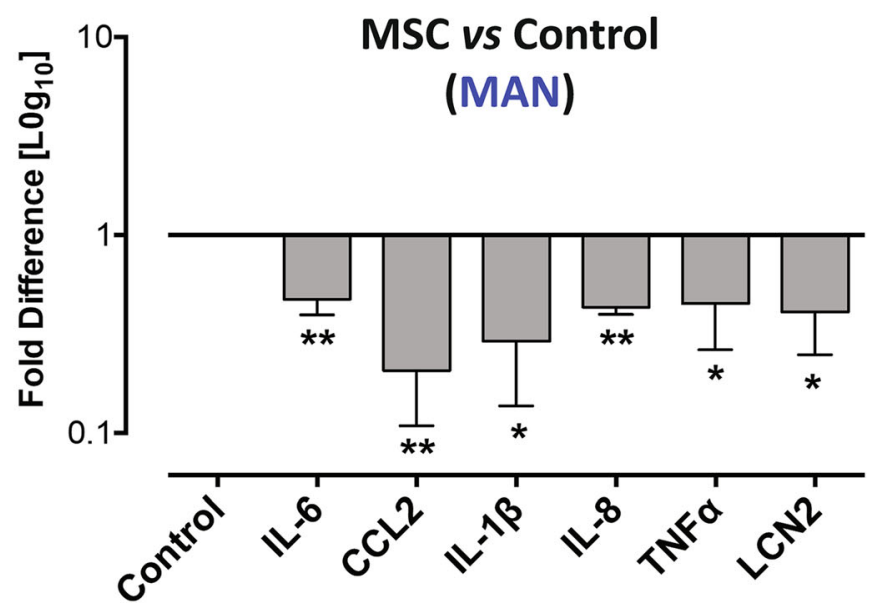

Fig. 7 Validation of the effect of BM-MSC on RPTEC-TERT1 expression of inflammation-related transcripts. Graphical representations of qRT-PCR analysis of the relative expression of RNA transcripts for IL-6, MCP-1 (CCL2), IL-1 $\beta$, IL-8, TNFa and NGAL (LCN2) in RPTEC/TERT1 cell monolayers cultured for 5 days in high glucose (HG) (a) or mannitol (MAN) (b) in the presence of BM-MSC-containing transwells (MSC) or no cells (control) for the final 2 days of culture. Results are expressed as mean \pm SD for $n=3$ samples per condition of the fold difference for MSC vs. control as determined relative to the housekeeping gene RPLPO using the $2^{-\Delta \Delta \mathrm{Ct}}$ method. Statistical analysis by Student's unpaired $t$ test, ${ }^{*} p<0.01,{ }^{*} p<0.05$, ns $=$ not significant

in culture in macrophage medium alone for a further 24 $\mathrm{h}$, following which production of pro-inflammatory cytokines and chemokines was quantified by ELISA of the macrophage culture supernatants. Macrophages cultured for $24 \mathrm{~h}$ in the absence and presence of a stimulatory cocktail of LPS, IFN $\gamma$ and TNF $\alpha$ served as additional controls. As shown in Fig. 8b, primary human macrophages showed significant elevation in the levels of those cytokines/chemokines when exposed for $24 \mathrm{~h}$ to $\mathrm{CM}$ from RPTEC/TERT1 cells under HG compared to MAN condition. When macrophages were exposed to $\mathrm{CM}$ from the equivalent RPTEC/TERT1-MSC co-cultures, reduced secretion of IL-8, MCP-1, IL-6 and TNF $\alpha$ was observed-being most significant for IL- 8 and TNF $\alpha$. The magnitude of induction of IL-8, MCP-1 and TNF $\alpha$ following macrophage exposure to HG-CM from RPTEC alone was comparable to that induced by stimulation with LPS, IFN $\gamma$ and TNFa. The results of qRT-PCR performed on RNA extracted from macrophages at the end of the experiment were inconclusive due to variability across technical replicates (data not shown). It was not possible, therefore, to confirm whether reduced macrophage secretion of inflammatory mediators was mediated specifically through suppression of transcription. A conceptual model for MSC modulation of HG-induced RPTEC/macrophage cross-talk based on the experimental results of the study is shown in Fig. 8c.

\section{Discussion}

A growing body of evidence indicates that RPTEC play an important role in the pathogenesis of DKD [17, 21, $25,39-41]$. In vitro observations using immortalised cell lines such as HK-2 provide a valuable, simplified system in which to explore specific effects of the diabetic milieu 

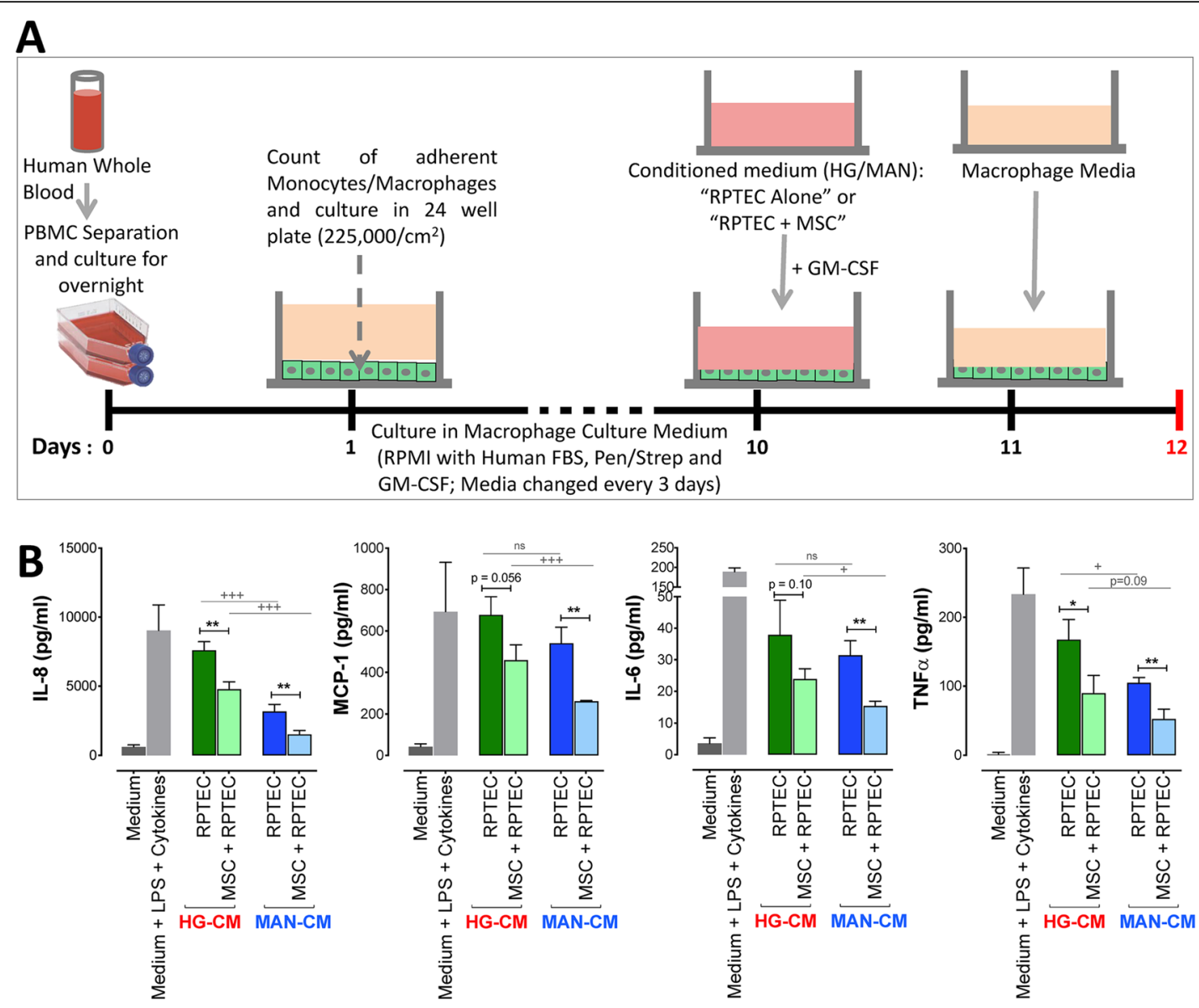

C
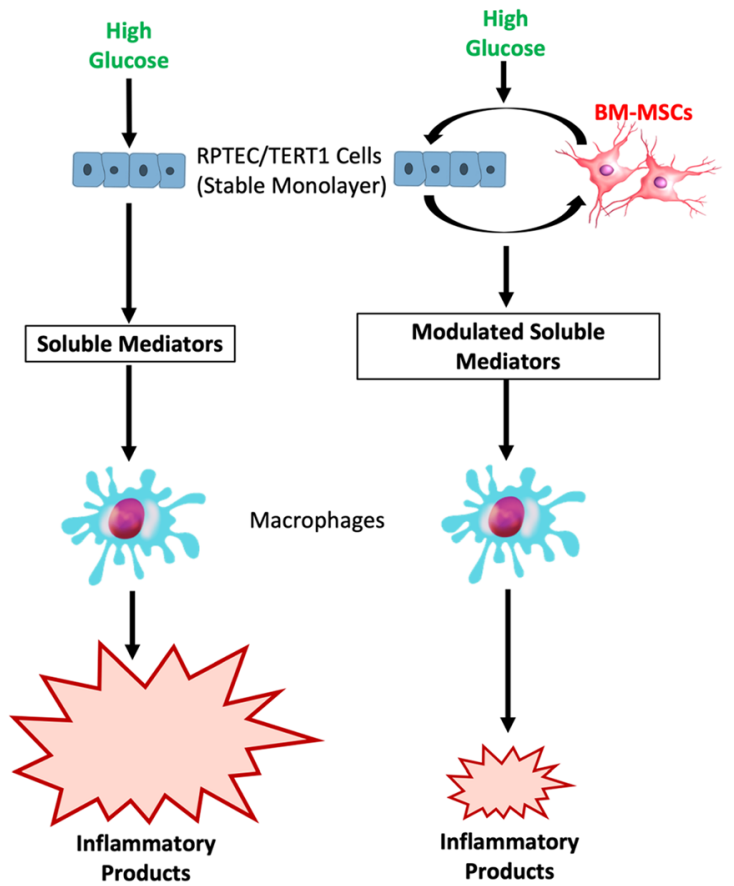

Fig. 8 (See legend on next page.) 


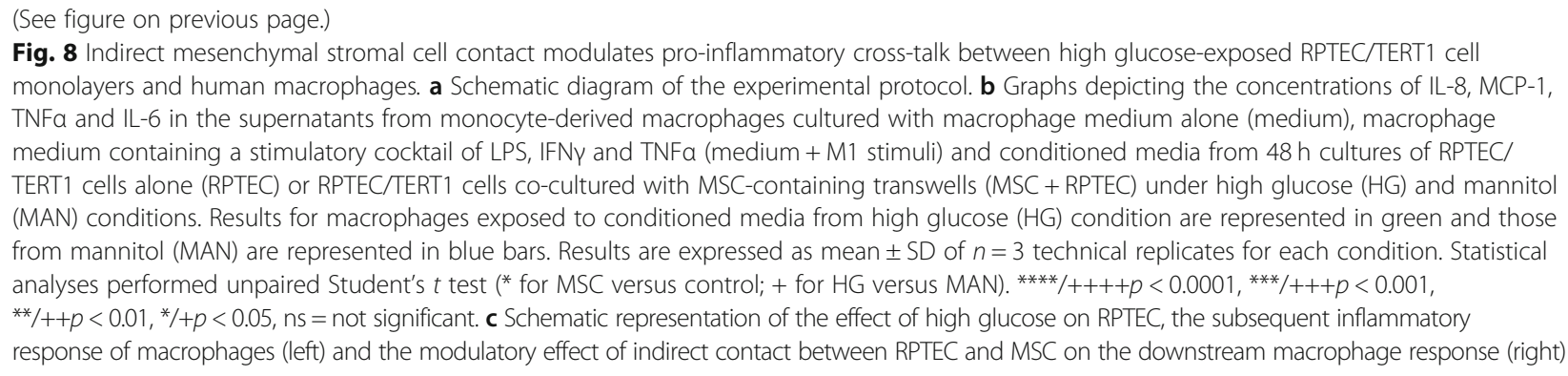

on renal epithelial cell biology but have limitations when comparing with primary RPTEC. Recent studies suggest that stable monolayers of RPTEC/TERT1 cells provide superior phenotypic and functional comparability to primary tubular epithelium [24-27]. In our hands, in keeping with previous reports [24-27], RPTEC/TERT1 cells formed stable monolayers after 12 days with an epithelial-like, cobblestone morphology which remained stable following a further 5-day exposure to HG or MAN. In this system, we observed that prolonged HG exposure induced a heightened inflammatory phenotype characterised by progressively increasing secretion of IL6, IL-8 and MCP-1 along with greater release of the tubular injury biomarker NGAL. For IL-6 and MCP-1, our observations are comparable to those of Tang et al. in confluent, growth-arrested primary RPTEC, albeit only becoming evident compared to normal glucose and MAN controls following a longer exposure time [42]. Importantly, although RNA-seq analysis revealed a range of other gene expression changes, we did not observe overt toxicity or evidence of loss of epithelial-like morphology of the monolayers during the period of exposure to HG or MAN.

Consistent with the HG-induced increase in IL-6 secretion by RPTEC/TERT1 monolayers, patients with DKD have increased renal IL-6 expression which correlates with kidney hypertrophy and albuminuria [43, 44] and increases with stage of DKD [45]. Exposure to HG for $96 \mathrm{~h}$ or more also resulted in increased secretion of IL- 8 by RPTEC/TERT1 cells. Of interest, Tashiro et al. reported increased levels of IL-8 in urine samples from the patients with early-stage DKD [46] while others observed increased IL- 8 production by tubular epithelial cells via activation of NF- $\mathrm{kB}, \mathrm{ERK} 1 / 2$ and STAT1 signalling in a glycated-albumin-induced diabetes model [47]. Similarly, NF-кB-dependent upregulation of MCP-1 in albumin-treated RPTEC and increased MCP-1 expression in kidney biopsy samples from patients with DKD have been reported $[48,49]$. In a hyperglycaemic environment, increased MCP-1 production by mesangial and tubular epithelial cells has also been observed in vitro $[14,50]$. Functionally, tubular cell-derived MCP-1 triggers infiltration of the interstitium by monocytes which, along with resident macrophages, secrete additional proinflammatory cytokines [16]. We also describe here a progressive increase in the release of NGAL, a comparatively new biomarker of DKD, following exposure of RPTEC/ TERT1 stable monolayers to $\mathrm{HG}$ for $80 \mathrm{~h}$ or more. Of relevance, NGAL is elevated in serum and urine of DKD patients [51-53]. Furthermore, Nielsen et al. demonstrated an association between urinary NGAL and rate of eGFR decline with the implication that renal tubular cells represent the major source of the urinary NGAL release [52]. Taken together, these observations support the relevance of our findings in the RPTEC/TERT1 culture system to renal interstitial inflammatory events related to hyperglycaemia and diabetes.

Human bone marrow-derived MSC produce immunomodulatory and cytoprotective mediators that act in a paracrine manner on a range of target cells to downregulate the production of pro-inflammatory cytokines and inhibit inflammatory signalling pathways [36, 54-56]. In animal models of diabetes, hBM-MSC infusions reduced matrix deposition in the mesangium [54] and glomerulus [56]. Nagaishi et al. studied the effects of BM-MSC treatment in insulin-deficient and insulin-resistant DKD models and demonstrated the benefits following systemic administration of both cells and CM [37]. Similarly, Lv et al. reported reduced intra-renal expression of IL1- $\beta$, IL-6, TNF $\alpha$ and MCP-1 following intravenous injection of MSC in a streptozotocin-induced rat model of DKD [55]. The reduction in MCP-1 and other inflammatory cytokines was associated with decreased macrophage infiltration and reduced severity of renal structural injury. In keeping with these in vivo findings, our experiments to evaluate the effect of human BMMSC on human RPTEC/TERT1 cells in the setting of prolonged HG exposure revealed anti-inflammatory effects both of MSC-CM and of indirect (transwell) coculture of RPTEC-TERT1 cells with MSC-with the latter being more potent. To our knowledge, this is the first study to show the effect of MSC and their soluble products on RPTEC/TERT1 cells as an in vitro model of diabetic proximal tubulopathy. The results indicate that MSC have the potential to modulate RPTEC dysfunction in DKD either from a distant anatomical site or locally 
within the kidney. Perhaps surprisingly, MSC-CM depleted of EV had comparable anti-inflammatory effects to those of EV-containing CM, indicating that the paracrine anti-inflammatory effects were unlikely to be mediated by MSC-derived EV despite evidence that they may have reno-protective properties $[57,58]$. Whether purified MSC-EV may have a distinct modulatory effect on HG-induced inflammatory responses of RPTEC/TERT1 monolayers and, if so, what mechanisms underlie such an effect remain an interesting question that merits further investigation.

Although knowledge of the mechanism of action of MSC in vivo is incomplete, the paracrine effect of a range of inducible factors is well established. Transcriptomic analysis of RPTEC/TERT1 cell monolayers demonstrated that prolonged HG exposure was associated with gene expression changes that were enriched for biological processes and signalling pathways of relevance to immune response and inflammation. We also observed differential gene expression between MAN and CTRL culture conditions that likely reflect the influence of increased osmolality on RPTEC-TERT1 monolayers. However, the findings that higher numbers of DEGs were identified for HG vs CTRL than for MAN vs CTRL and that the HG condition separated fully from CTRL and MAN on a principal component analysis of the RNA-seq data are in keeping with a distinct effect of HG rather than a non-specific effect of increased osmolality. Interestingly, under both HG and MAN conditions, the modulatory effects of indirect coculture with MSC on the RPTEC-TERT1 monolayer transcriptome were also enriched for immune/inflammatory pathways-with the TNF signalling pathway being particularly prominent. Modulation of the HG-induced TNF signalling pathway following MSC co-culture included reversion toward control levels of the increased expression of genes related to leukocyte recruitment (CCL2/MCP-1, CXCL1, CXCL2, CXCL3), leukocyte activation (CSF-2), inflammatory cytokines (IL-1 $\beta$, IL-6) and cell adhesion (ESelectin). Results for several inflammatory mediators/ products (IL-6, IL-8, CCL2/MCP-1 and LCN2/NGAL) were consistent across RNA-seq, qRT-PCR and ELISA. An overall implication of these results is that human MSC, delivered directly to the kidney or transmigrating to renal interstitial spaces in the setting of diabetes/hyperglycemia, are likely to initiate a paracrine interaction with RPTEC that potently downregulates chronic inflammatory signalling. Furthermore, we provide evidence that the modulatory effect of MSC on release of inflammatory mediators by RPTEC in the setting of HG may have downstream effects on the response of monocyte-derived macrophages which are known to infiltrate the kidney and mediate renal interstitial damage during DKD [31]. Inflammatory stimuli to RPTEC have been shown to result in cross-talk with intra-renal macrophages through a number of mechanisms including cytokine/chemokine secretion, transfer of bioactive molecules in extracellular vesicles, release of danger-associated molecular patterns and triggering of specific forms of necrotic cell death [59-61].

A relatively large body of literature exists in support of the potential benefits of MSC to slow the progression of DKD through anti-inflammatory mechanisms [2]. To date, however, only one early-phase clinical trial of a stromal cell therapy has been completed in human subjects with DKD. This demonstrated that, in relatively advanced DKD due to type $2 \mathrm{DM}$, intravenous injection of allogeneic bone marrow-derived $\mathrm{Stro}^{+}$mesenchymal precursor cells was safe up to 24 weeks postadministration and was associated with preliminary evidence of efficacy including decreased serum IL-6 compared to placebo [62].

In addition to the obvious caveat that experimental work conducted in vitro using a cell line will require validation in more physiologically relevant systems, some specific limitations of the study must be acknowledged. Firstly, while our culture conditions and experimental durations are quite comparable to those used by other investigators who have extensively characterised RPTEC/ TERT1 cell epithelial monolayer formation [26], they cannot be said to be identical. Thus, the influence of variability in culture conditions on the physiological relevance of our experimental findings cannot be fully determined. Nonetheless, it is clear that, in our hands, the cells consistently generated typical, mature epithelial-like monolayers and that subsequent 4-5-day exposure of the monolayers to HG and MAN did not result in overt cellular toxicity or transformation to a nonepithelial phenotype. Lack of elucidation of a clear intracellular signalling mechanism to explain HG-induced changes in the expression and secretion of inflammatory mediators by RPTEC/TERT1 cell monolayers (and its suppression by factors released by MSC) is a second limitation. While involvement of alternative signalling pathways, of post-transcriptional/post-translation modification of the gene products or of altered intracellular trafficking/secretion of inflammatory mediators may explain the observations, it is also possible that experiments carried out at earlier or later time-points could have revealed increased activity of one or more predicted signalling pathways under HG conditions. Thirdly, while our experimental focus for the study was on HGinduced inflammatory response of RPTECT/TERT1 monolayers and its modulation by MSC, other potentially important aspects of the altered transcriptomic profiles revealed by RNA-seq have not been functionally validated and explored. By sharing the full lists of DEG we have identified under different experimental conditions, we anticipate that these data can be further 
exploited through deeper functional investigation by others in the field. Finally, a limitation of our experiments involving the transfer of conditioned media from RPTEC/MSC co-cultures to primary macrophages is that they do not exclude the possibility that MSC- and RPTEC/TERT1-derived soluble mediators act on macrophages independently of each other rather than as a result of a distinctive MSC/ RPTEC cross-talk. Thus, additional experiments beyond the scope of the current study will be needed to dissect the individual mechanistic contributions of RPTEC and MSC on downstream activity of macrophages and to investigate the potency of MSC to regulate RPTEC signalling to macrophages across a range of pathogenic conditions.

\section{Conclusions}

Our current study reveals a predominantly proinflammatory effect of prolonged HG exposure on stable monolayers of the RPTEC/TERT1 cell line during a 5day time window that is substantially modulated at a transcriptional level by soluble products of human MSC-particularly when the two cell types were cultured in close proximity. Experimentally, we also show that the combined secretome of RPTEC/MSC cocultures has the capacity to dampen macrophage inflammatory response under HG conditions. These results provide a novel platform for better understanding antiinflammatory mechanisms of action of MSC in in vivo studies and clinical trials of DKD. Our in vitro system also has the potential for identifying new targets of intervention for diabetes-associated proximal tubulopathy and pro-inflammatory epithelial cell/macrophage crosstalk. Further studies are also needed to elucidate the mechanisms whereby HG exposure induces a prolonged increased secretion of cytokines, chemokines and markers of inflammation by RPTEC in the absence of persistent over-activity of NF-kB, MAPK and other expected intracellular signalling pathways.

\section{Supplementary information}

Supplementary information accompanies this paper at https://doi.org/10. 1186/s13287-019-1424-5.

\footnotetext{
Additional file 1. Supplementary methods document.

Additional file 2: Table S1. List of primer sequences used for GRT-PCR. Additional file 3: Figure S1. Effect of High D-Glucose on RPTEC/TERT1 Cells. A. Effect of seeding density on growth of RPTEC-TERT-1 cells. Here, three different cell numbers per square centimeter $\left(\mathrm{cm}^{2}\right)$, labelled as Low (12500 cells $/ \mathrm{cm}^{2}$ ), Medium (12500 cells/ $\mathrm{cm}^{2}$ ) and High (12500 cells/cm²), are represented as Blue, Green and Grey coloured lines respectively. Cells grown in 24 well plate for different time points and number of cells counted by haemocytometer using trypan blue and represented as cells/ $\mathrm{cm}^{2}$. B. Effect of high glucose on growth of RPTEC-TERT-1. Cells were seeded at $27500 / \mathrm{cm}^{2}$. and counted at different time points using haemocytometer. C. Downstream experimental plan. RPTEC-TERT-1 cells cultured at $27500 / \mathrm{cm}^{2}$, media replaced every second day. From day $12,25 \mathrm{mM}$ of D-Glucose or D-Mannitol (osmotic control to glucose) was administered for different time periods Effect of high Glucose on RPTECs for
}

downstream comparisons of glucose versus controls: formation of stable monolayer by microscope; D. Similar cell size confirmed by flow cytometry.

Additional file 4: Figure S2. Combined effect of high glucose and albumin on RPTEC/TERT1 inflammatory responses. A. Schematic diagram of the experimental protocol. In brief, RPTEC-TERT-1 cells cultured at $27500 / \mathrm{cm}^{2}$, medium was replaced every second day. From day 12 , cells were grown in high-glucose or control conditions (CTRL/HG/MAN) with or without $100 \mu \mathrm{g} / \mathrm{ml}$ human serum albumin. Mediium was replaced at day 15 for a further two days. B. Mean \pm SD levels of inflammatory mediators including IL-8 (top left), IL-6 (top right), MCP-1 (bottom left) and NGAL (bottom right) in the supernatants are represented in grey (CTRL), blue (HG) and green (MAN) bars. Bright colours represent the levels in samples when treated without albumin. * denoted unpaired t-tests for CTRL vs HG, HG vs MAN, MAN vs CTRL. $¥$ denoted ANOVA to analyse differences between CTRL, HG and MAN.

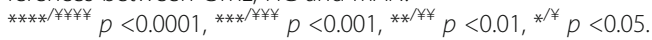

Additional file 5: Figure S3. Combined effect of high glucose and IL$1 \beta$ as inflammatory cytokine stimuli on RPTEC/TERT1 responses. A. Schematic diagram of the experimental protocol. In brief, RPTEC/TERT1 cells were cultured at $27500 / \mathrm{cm}^{2}$, medium was replaced every second day. From day 12 , cells were grown in high-glucose or control conditions (CTRL/HG/MAN). Medium was replaced at day-15. In addition to CTRL/HG/MAN, cells were treated with- or without- $1 \mathrm{ng} / \mathrm{ml} \mathrm{IL}-1 \beta$ for the final two days; B. Mean \pm SD levels of inflammatory mediators including IL-8 (top left), IL-6 (top right), MCP-1 (bottom left) and NGAL (bottom right) in the supernatant samples represented in grey (CTRL), blue (HG) and green (MAN) bars. Bright colours represent the levels in samples when treated without IL-1 $\beta .{ }^{*}$ denoted unpaired t-tests for CTRL vs HG, HG vs MAN, and MAN vs CTRL. $¥$ denoted ANOVA to test for differences between $C T R L, H G$ and MAN.

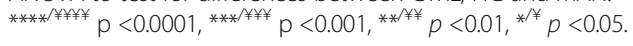

Additional file 6: Figure S4. A: Exposure of RPTEC/TERT1 cells to highGlucose did not alter expression in any common inflammatory signalling molecules. RPTEC-TERT-1 cells were cultured at $27500 / \mathrm{cm}^{2}$, medium was replaced every second day. From day 12, cells were grown in highglucose or control conditions (CTRL/HG/MAN) for 24, 48 and 96 hours. Using western blotting, cell pellets were harvested for investigating the expressions of different signalling proteins including: total and phosphorylated forms of p65 NFkB (nuclear factor kappa B - p65 sub unit), p38 MAPK (P38 mitogen-activated protein kinase), ERK-1/2 (extracellular signal-regulated kinase 1/2), STAT-1 (Signal transducer and activator of transcription 1), PKCa (Protein kinase C alpha) and total PPAR- $($ Peroxisome proliferator-activated receptor gamma) as well as housekeeping protein $\beta$-Actin (Beta Actin).

Additional file 7: Figure S4. B: Semi-quantitative analyses of the western blots as in Figure S4A. ImageJ software was used to perform semi-quantitative analysis of the blots. The area and its corresponding percentage of blots were calculated. Densitometric data were then normalized for the housekeeping protein followed by further normalization relative to the control. Statistical analyses were performed using GraphPad prism. Results were expressed as the Mean \pm SD for three technical replicates per condition. $p$ values $\leq 0.05$ were considered significant at: ${ }^{*} p<0.05,{ }^{* *} p<0.01,{ }^{* * *} p<0.001$

Additional file 8: Figure S5. Human bone-marrow derived MSCS showed its cell surface characteristics and were able to differentiate. A. Immunophenotyping of cultured human BM-MSCs for different specific cell surface markers by flow cytometry. B. Osteogenic and adipogenic differentiation capacity of BM-MSCs. Unpaired t test with Welch's correction ${ }^{*} \mathrm{p}<0.05 ;{ }^{* *} \mathrm{p}<0.01 ;{ }^{* * *} \mathrm{p}<0.001$.

Additional file 9: Table S2. List of DEGs with significant Fold Changes in High-Glucose vs Mannitol.

Additional file 10: Table S3. List of DEGs with significant Fold Changes in High-Glucose vs Control.

Additional file 11: Table S4. List of DEGs with significant Fold Changes in Mannitol vs Control.

Additional file 12: Figure S6. Involvement of biological processes and signalling pathways associated with differentially expressed genes in high-glucose exposed RPTEC/TERT1 cells. (A) Pathway Enrichment analysis 
of differentially expressed genes (DEGs) performed based on KEGG database; (B) Scatter plot for the top 20 pathways of KEGG enrichment results. Here, $X$ axis represents number of DEGs. $Y$ axis represents second KEGG pathway terms. All second pathway terms were grouped in top pathway terms indicated in different colour.

Additional file 13: Figure S7. Up-regulation of genes for inflammatory cytokines following exposure of RPTEC/TERT1 cells to high-glucose (TNFSignalling pathway is a representative example). Up-regulated genes are marked with red borders. Unchanged genes are marked with black borders.

Additional file 14: Table S5. List of DEGs with significant Fold Changes in RPTEC/TERT1 cells without and with MSC co-culture in high glucose (HG) condition.

Additional file 15: Table S6. List of DEGs with significant Fold Changes in RPTEC/TERT1 cells without and with MSC co-culture in mannitol (MAN) condition.

Additional file 16: Figure S8. Differential Expression of Genes in Presence of Indirect Contact of MSCs. Pathway Enrichment Analysis of Differentially Expressed Genes (DEGs) performed based on KEGG database by pairwise analysis. Here, $X$ axis represents number of DEGs. Y axis represents second KEGG pathway terms. All second pathway terms were grouped in top pathway terms indicated in different colour.

Additional file 17: Figure S9. TNF-Signalling pathway as a representative example of the signalling pathways involved in MSC mediated antiinflammatory effects (in high-glucose environment). Here, downregulated genes are marked with green borders and unchanged genes are marked with black borders.

\section{Abbreviations}

AGE: Advanced glycation end-products; BM-MSC: Bone marrow-derived mesenchymal stromal cells; CM: Conditioned medium; CTRL: Control; DEG: Differentially expressed gene; DKD: Diabetic kidney disease; DM: Diabetes mellitus; DMEM: Dulbecco's modified Eagle medium: ESRD: End-stage renal disease; EV: Extracellular vesicle; FCS: Foetal calf serum; GM-CSF: Granulocyte macrophage colony stimulating factor; HCEC: Human corneal endothelial cell; HG: High glucose; HSA: Human serum albumin; hTERT: Human telomerase reverse transcriptase; IFNY: Interferon gamma; IL: Interleukin; MAN: Mannitol; MCP-1: Monocyte chemoattractant protein 1; MSC: Mesenchymal stromal cell; NGAL: Neutrophil gelatinase-associated lipocalin; PI: Propidium iodide; RIN: RNA integrity number; RNA-seq: RNA sequencing; ROS: Reactive oxygen species; RPTEC: Renal proximal tubular epithelial cell; TNFa: Tumour necrosis factor alpha

\section{Acknowledgements}

All flow cytometry experiments were performed in the NUI Galway Flow Cytometry Core Facility which is supported by funds from NUI Galway, Science Foundation Ireland, the Irish Government's Programme for Research in Third Level Institutions, Cycle 5 and the European Regional Development Fund. The materials presented and views expressed here are the responsibility of the author(s) only. Human corneal endothelial cells were kindly provided by Prof. Thomas Ritter, REMEDI, School of Medicine, NUI Galway. We wish to acknowledge the generous assistance of Dr. Paula O'Shea, Clinical Biochemistry Laboratory, Galway University Hospitals for facilitating the analysis of culture supernatant osmolality.

\section{Authors' contributions}

$\mathrm{MNI}$ contributed to the conception and design, collection and/or assembly of data, data analysis and interpretation, manuscript writing, and final approval of the manuscript. TPG contributed to the conception and design, collection and/or assembly of data, data analysis and interpretation, and final approval of the manuscript. ES contributed to the collection and/or assembly of data, data analysis and interpretation, and final approval of the manuscript. SR contributed to the collection and/or assembly of data, data analysis and interpretation, and final approval of the manuscript. JQ contributed to the collection and/or assembly of data, data analysis and interpretation, and final approval of the manuscript. JC contributed to the collection and/or assembly of data and final approval of the manuscript. JMCC contributed to the provision of study material and final approval of the manuscript. TMcM contributed to the conception and design, provision of study material, data analysis and interpretation, and final approval of manuscript. MDG contributed to the conception and design, financial support, data analysis and interpretation, manuscript writing, and final approval of manuscript.

\section{Funding}

The research was supported by a grant from the European Commission [Horizon 2020 Collaborative Health Project NEPHSTROM (grant number 634086; TPG, MNI, MDG)]. Other funding sources that contributed to the work were grants from the European Commission [FP7 Collaborative Health Project VISICORT (grant number 602470; MDG, JC)], from Science Foundation Ireland [REMEDI Strategic Research Cluster (grant number 09/SRC-B1794; MDG) and CÚRAM Research Centre (grant number 13/RC/2073; MDG)], from the Health Research board of Ireland (grant number HRA_POR/2013/341; JC, MDG) and the European Regional Development Fund. TPG is supported by a Hardiman Scholarship from the College of Medicine, Nursing and Health Science, National University of Ireland Galway and a bursary from the Irish Endocrine Society/Royal College of Physicians of Ireland. JMCC and TMCM are funded by Science Foundation Ireland (grant number 12/IP/1686) and by the School of Biomolecular and Biomedical Science, University College Dublin. The EU Commission takes no responsibility for any use made of the information set out.

\section{Availability of data and materials}

RNA-seq data will be made publically available through GEO upon publication. All other datasets used and/or analysed during the current study are available from the corresponding author on reasonable request.

\section{Ethics approval and consent to participate}

Human healthy volunteer bone marrow samples were sourced through the HRB Clinical Research Facility Galway according to a protocol approved by the Clinical Research Ethics Committee of the Galway University Hospitals. Primary culture expansion of MSC from bone marrow samples was performed at the Centre for Cell Manufacturing Ireland, National University of Ireland Galway.

\section{Consent for publication}

Not applicable.

\section{Competing interests}

The authors declare that they have no competing interests.

\section{Author details}

${ }^{1}$ Regenerative Medicine Institute (REMEDI) at CÚRAM Centre for Research in Medical Devices, School of Medicine, National University of Ireland Galway, Galway, REMEDI, Biomedical Sciences, Corrib Village, Dangan, Galway H91 TK33, Ireland. ${ }^{2}$ Centre for Endocrinology, Diabetes and Metabolism, Galway University Hospitals, Galway, Ireland. ${ }^{3}$ School of Biomolecular and Biomedical Science, Conway Institute, University College Dublin, Dublin, Ireland.

Received: 16 May 2019 Revised: 8 September 2019

Accepted: 24 September 2019 Published online: 19 November 2019

\section{References}

1. Thomas MC, Cooper ME, Zimmet P. Changing epidemiology of type 2 diabetes mellitus and associated chronic kidney disease. Nat Rev Nephrol. 2015;12:73-81.

2. Griffin TP, Martin WP, Islam N, et al. The promise of mesenchymal stem cell therapy for diabetic kidney disease. Curr Diab Rep. 2016;16:42.

3. Alicic RZ, Rooney MT, Tuttle KR. Diabetic kidney disease: challenges, progress, and possibilities. Clin J Am Soc Nephrol. 2017;12:2032-45.

4. Afkarian M, Sachs MC, Kestenbaum B, et al. Kidney disease and increased mortality risk in type 2 diabetes. J Am Soc Nephrol. 2013;24:302-8.

5. Bloomgarden ZT. Inflammation and insulin resistance. Diabetes Care. 2003; 26:1922-6.

6. Dandona P, Aljada A, Bandyopadhyay A. Inflammation: the link between insulin resistance, obesity and diabetes. Trends Immunol. 2004:25:4-7.

7. Navarro-Gonzalez JF, Mora-Fernandez C, Muros de Fuentes M, et al. Inflammatory molecules and pathways in the pathogenesis of diabetic nephropathy. Nat Rev Nephrol. 2011;7:327-40. 
8. Lim AKH, Tesch GH. Inflammation in diabetic nephropathy. Mediat Inflamm. 2012;2012:146-54.

9. García-García PM, Getino-Melián MA, Domínguez-Pimentel V, et al. Inflammation in diabetic kidney disease. World J Diabetes. 2014;5:431-43.

10. Ha H, Yu MR, Choi YJ, et al. Role of high glucose-induced nuclear factorkappaB activation in monocyte chemoattractant protein-1 expression by mesangial cells. J Am Soc Nephrol. 2002;13:894-902.

11. Morii T, Fujita H, Narita T, et al. Association of monocyte chemoattractant protein-1 with renal tubular damage in diabetic nephropathy. J Diabet Complicat. 2003;17:11-5.

12. Kanamori H, Matsubara T, Mima A, et al. Inhibition of MCP-1/CCR2 pathway ameliorates the development of diabetic nephropathy. Biochem Biophys Res Comm. 2007;360:772-7.

13. Chow FY, Nikolic-Paterson DJ, Ma FY, et al. Monocyte chemoattractant protein-1-induced tissue inflammation is critical for the development of renal injury but not type 2 diabetes in obese $\mathrm{db} / \mathrm{db}$ mice. Diabetologia. 2007:50:471-80

14. Wada T, Yokoyama H, Matsushima K, et al. Monocyte chemoattractant protein-1: does it play a role in diabetic nephropathy? Nephrol Dial Transplant. 2003;18:457-9.

15. Viedt C, Dechend R, Fei J, et al. MCP-1 induces inflammatory activation of human tubular epithelial cells: involvement of the transcription factors, nuclear factor-KB and activating protein-1. J Am Soc Nephrol. 2002;13:1534-47.

16. Sun L, Kanwar YS. Relevance of TNF-alpha in the context of other inflammatory cytokines in the progression of diabetic nephropathy. Kidney Int. 2015;88:662-5.

17. Tang SC, Lai KN. The pathogenic role of the renal proximal tubular cell in diabetic nephropathy. Nephrol Dial Transplant. 2012;27:3049-56.

18. Wolf $\mathrm{G}$, Neilson EG, Goldfarb $\mathrm{S}$, et al. The influence of glucose concentration on angiotensin II-induced hypertrophy of proximal tubular cells in culture. Biochem Biophys Res Comm. 1991;176:902-9.

19. Allen DA, Harwood S, Varagunam M, et al. High glucose-induced oxidative stress causes apoptosis in proximal tubular epithelial cells and is mediated by multiple caspases. FASEB J. 2003;17:908-10.

20. Jenkin KA, McAinch AJ, Zhang Y, et al. Elevated cannabinoid receptor 1 and $\mathrm{G}$ protein-coupled receptor 55 expression in proximal tubule cells and whole kidney exposed to diabetic conditions. Clin Exp Pharmacol Physiol. 2015:42:256-62

21. Gilbert RE. Proximal tubulopathy: prime mover and key therapeutic target in diabetic kidney disease. Diabetes. 2017:66:791-800.

22. Holm J, Hemmingsen L, Nielsen NV. Low-molecular-mass proteinuria as a marker of proximal renal tubular dysfunction in normo- and microalbuminuric non-insulin-dependent diabetic subjects. Clin Chem. 1993;39:517-9.

23. Panchapakesan U, Pegg K, Gross S, et al. Effects of SGLT2 inhibition in human kidney proximal tubular cells--renoprotection in diabetic nephropathy? PLoS One. 2013;8:-e54442.

24. Wieser M, Stadler G, Jennings P, et al. hTERT alone immortalizes epithelial cells of renal proximal tubules without changing their functional characteristics. Am J Physiol Renal Physiol. 2008;295:F1365-75.

25. Slyne J, Slattery C, McMorrow T, et al. New developments concerning the proximal tubule in diabetic nephropathy: in vitro models and mechanisms. Nephrol Dial Transplant. 2015;30(Suppl 4):iv60-7.

26. Aschauer $L$, Gruber $L N$, Pfaller $W$, et al. Delineation of the key aspects in the regulation of epithelial monolayer formation. Mol Cell Biol. 2013;33:2535-50.

27. Ellis JK, Athersuch TJ, Cavill R, et al. Metabolic response to low-level toxicant exposure in a novel renal tubule epithelial cell system. Mol BioSyst. 2011;7:247-57

28. Galkina E, Ley K. Leukocyte recruitment and vascular injury in diabetic nephropathy. J Am Soc Nephrol. 2006;17:368-77.

29. Chow F, Ozols E, Nikolic-Paterson DJ, et al. Macrophages in mouse type 2 diabetic nephropathy: correlation with diabetic state and progressive renal injury. Kidney Int. 2004;65:116-28.

30. Chow FY, Nikolic-Paterson DJ, Atkins RC, et al. Macrophages in streptozotocin-induced diabetic nephropathy: potential role in renal fibrosis. Nephrol Dial Transplant. 2004;19:2987-96.

31. Nguyen D, Ping F, Mu W, et al. Macrophage accumulation in human progressive diabetic nephropathy. Nephrology. 2006;11:226-31.

32. Tan TK, Zheng G, Hsu TT, et al. Matrix metalloproteinase-9 of tubular and macrophage origin contributes to the pathogenesis of renal fibrosis via macrophage recruitment through osteopontin cleavage. Lab Investig. 2013; 93:434-49.
33. Griffin MD, Elliman SJ, Cahill E, et al. Concise review: adult mesenchymal stromal cell therapy for inflammatory diseases: how well are we joining the dots? Stem Cells. 2013;31:2033-41.

34. Uccelli A, Moretta L, Pistoia V. Mesenchymal stem cells in health and disease. Nat Rev Immunol. 2008;8:726.

35. Dominici M, Le Blanc K, Mueller I, et al. Minimal criteria for defining multipotent mesenchymal stromal cells. The International Society for Cellular Therapy position statement. Cytotherapy. 2006;8:315-7.

36. Abdel Aziz MT, Wassef MA, Ahmed HH, et al. The role of bone marrow derived-mesenchymal stem cells in attenuation of kidney function in rats with diabetic nephropathy. Diabetol Metab Syndr. 2014;6:34.

37. Nagaishi K, Mizue Y, Chikenji T, et al. Mesenchymal stem cell therapy ameliorates diabetic nephropathy via the paracrine effect of renal trophic factors including exosomes. Sci Rep. 2016;6:34842.

38. Moghadasali R, Mutsaers HA, Azarnia M, et al. Mesenchymal stem cellconditioned medium accelerates regeneration of human renal proximal tubule epithelial cells after gentamicin toxicity. Exp Toxicol Pathol. 2013;65:595-600.

39. Xing L, Song E, Yu CY, et al. Bone marrow-derived mesenchymal stem cells attenuate tubulointerstitial injury through multiple mechanisms in UUO model. J Cell Biochem. 2019;120:9737-46.

40. Vallon V, Thomson SC. Renal function in diabetic disease models: the tubular system in the pathophysiology of the diabetic kidney. Annu Rev Physiol. 2012;74:351-75.

41. Reidy K, Kang HM, Hostetter T, et al. Molecular mechanisms of diabetic kidney disease. J Clin Invest. 2014;124:2333-40.

42. Tang SC, Chan LY, Leung JC, et al. Bradykinin and high glucose promote renal tubular inflammation. Nephrol Dial Transplant. 2010;25:698-710.

43. Navarro JF, Milena FJ, Mora C, et al. Renal pro-inflammatory cytokine gene expression in diabetic nephropathy: effect of angiotensin-converting enzyme inhibition and pentoxifylline administration. Am J Nephrol. 2006;26: $562-70$.

44. Thomson SC, Deng A, Bao D, et al. Ornithine decarboxylase, kidney size, and the tubular hypothesis of glomerular hyperfiltration in experimental diabetes. J Clin Invest. 2001;107:217-24.

45. Saraheimo M, Teppo AM, Forsblom C, et al. Diabetic nephropathy is associated with low-grade inflammation in type 1 diabetic patients. Diabetologia. 2003;46:1402-7.

46. Tashiro K, Koyanagi I, Saitoh A, et al. Urinary levels of monocyte chemoattractant protein-1 (MCP-1) and interleukin-8 (IL-8), and renal injuries in patients with type 2 diabetic nephropathy. J Clin Lab Anal. 2002;16:1-4.

47. Tang SC, Leung JC, Chan LY, et al. Activation of tubular epithelial cells in diabetic nephropathy and the role of the peroxisome proliferator-activated receptor-gamma agonist. J Am Soc Nephrol. 2006;17:1633-43.

48. Wang $Y$, Rangan $G K$, Tay $Y C$, et al. Induction of monocyte chemoattractant protein-1 by albumin is mediated by nuclear factor kappaB in proximal tubule cells. J Am Soc Nephrol. 1999;10:1204-13.

49. Mezzano SA, Barria M, Droguett MA, et al. Tubular NF-kappaB and AP-1 activation in human proteinuric renal disease. Kidney Int. 2001;60:1366-77.

50. Tesch GH. MCP-1/CCL2: a new diagnostic marker and therapeutic target for progressive renal injury in diabetic nephropathy. Am J Physiol Renal Physiol. 2008;294:F697-701.

51. Bolignano D, Coppolino G, Lacquaniti A, et al. Neutrophil gelatinaseassociated lipocalin in the intensive care unit: time to look beyond a single, threshold-based measurement? Crit Care Med. 2009;37:2864.

52. Nielsen $\mathrm{SE}$, Reinhard $\mathrm{H}$, Zdunek $\mathrm{D}$, et al. Tubular markers are associated with decline in kidney function in proteinuric type 2 diabetic patients. Diabetes Res Clin Pract. 2012;97:71-6.

53. Chou KM, Lee CC, Chen CH, et al. Clinical value of NGAL, L-FABP and albuminuria in predicting GFR decline in type 2 diabetes mellitus patients. PLoS One. 2013;8:e54863.

54. Lee RH, Seo MJ, Reger RL, et al. Multipotent stromal cells from human marrow home to and promote repair of pancreatic islets and renal glomeruli in diabetic NOD/scid mice. Proc Natl Acad Sci U S A. 2006;103:17438-43.

55. Lv SS, Liu G, Wang JP, et al. Mesenchymal stem cells transplantation ameliorates glomerular injury in streptozotocin-induced diabetic nephropathy in rats via inhibiting macrophage infiltration. Int Immunopharmacol. 2013;17:275-82.

56. Ezquer F, Giraud-Billoud M, Carpio D, et al. Proregenerative microenvironment triggered by donor mesenchymal stem cells preserves renal function and structure in mice with severe diabetes mellitus. Biomed Res Int. 2015;2015:164703. 
57. He J, Wang $Y$, Lu X, et al. Micro-vesicles derived from bone marrow stem cells protect the kidney both in vivo and in vitro by microRNA-dependent repairing. Nephrology. 2015;20:591-600.

58. Grange C, lampietro C, Bussolati B. Stem cell extracellular vesicles and kidney injury. Stem Cell Invest. 2017:4:90.

59. Lv LL, Feng Y, Wen Y, et al. Exosomal CCL2 from tubular epithelial cells is critical for albumin-induced tubulointerstitial inflammation. J Am Soc Nephrol. 2018;29:919-35.

60. Mulay SR, Linkermann A, Anders HJ. Necroinflammation in kidney disease. J Am Soc Nephrol. 2016;27:27-39.

61. Lin M, Yiu WH, Wu HJ, et al. Toll-like receptor 4 promotes tubular inflammation in diabetic nephropathy. J Am Soc Nephrol. 2012;23:86-102.

62. Packham DK, Fraser IR, Kerr PG, et al. Allogeneic mesenchymal precursor cells (MPC) in diabetic nephropathy: a randomized, placebo-controlled, dose escalation study. EBioMedicine. 2016;12:263-9.

\section{Publisher's Note}

Springer Nature remains neutral with regard to jurisdictional claims in published maps and institutional affiliations.

Ready to submit your research? Choose BMC and benefit from:

- fast, convenient online submission

- thorough peer review by experienced researchers in your field

- rapid publication on acceptance

- support for research data, including large and complex data types

- gold Open Access which fosters wider collaboration and increased citations

- maximum visibility for your research: over $100 \mathrm{M}$ website views per year

At BMC, research is always in progress.

Learn more biomedcentral.com/submissions 